\title{
MAXIMAL LEFT IDEALS OF THE BANACH ALGEBRA OF BOUNDED OPERATORS ON A BANACH SPACE
}

\author{
H. G. DALES, TOMASZ KANIA, TOMASZ KOCHANEK, PIOTR KOSZMIDER, AND \\ NIELS JAKOB LAUSTSEN
}

\begin{abstract}
We address the following two questions regarding the maximal left ideals of the Banach algebra $\mathscr{B}(E)$ of bounded operators acting on an infinite-dimensional Banach space $E$ :

(I) Does $\mathscr{B}(E)$ always contain a maximal left ideal which is not finitely generated?

(II) Is every finitely-generated, maximal left ideal of $\mathscr{B}(E)$ necessarily of the form

$$
\{T \in \mathscr{B}(E): T x=0\}
$$

for some non-zero $x \in E$ ?

Since the two-sided ideal $\mathscr{F}(E)$ of finite-rank operators is not contained in any of the maximal left ideals given by $(*)$, a positive answer to the second question would imply a positive answer to the first.

Our main results are: (i) Question (I) has a positive answer for most (possibly all) infinite-dimensional Banach spaces; (ii) Question (II) has a positive answer if and only if no finitely-generated, maximal left ideal of $\mathscr{B}(E)$ contains $\mathscr{F}(E)$; (iii) the answer to Question (II) is positive for many, but not all, Banach spaces.
\end{abstract}

\section{INTRODUCTION AND STATEMENT OF MAIN RESUlTS}

The purpose of this paper is to study the maximal left ideals of the Banach algebra $\mathscr{B}(E)$ of (bounded, linear) operators acting on a Banach space $E$, particularly the maximal left ideals that are finitely generated. A general introduction to the Banach algebra $\mathscr{B}(E)$ can be found in $[9, \S 2.5]$. Our starting point is the elementary observation that $\mathscr{B}(E)$ always contains a large supply of singly-generated, maximal left ideals, namely

$$
\mathscr{M} \mathscr{L}_{x}=\{T \in \mathscr{B}(E): T x=0\} \quad(x \in E \backslash\{0\})
$$

(see Proposition 2.2 for details). We call the maximal left ideals of this form fixed, inspired by the analogous terminology for ultrafilters.

The Banach algebra $\mathscr{B}(E)$ is semisimple, as is well known (e.g., see [9, Theorem 2.5.8]); that is, the intersection of its maximal left ideals is $\{0\}$. We observe that this is already true for the intersection of the fixed maximal left ideals.

2010 Mathematics Subject Classification. Primary 47L10, 46H10; Secondary 47L20.

Key words and phrases. Finitely-generated, maximal left ideal; Banach algebra; bounded operator; inessential operator; Banach space; Argyros-Haydon space; Sinclair-Tullo theorem. 
In the case where the Banach space $E$ is finite-dimensional, an elementary result in linear algebra states that the mapping

$$
F \mapsto\{T \in \mathscr{B}(E): F \subseteq \operatorname{ker} T\}
$$

is an anti-isomorphism of the lattice of linear subspaces of $E$ onto the lattice of left ideals of $\mathscr{B}(E)$ (e.g., see [21, p. 173, Exercise 3]). Hence each maximal left ideal $\mathscr{L}$ of $\mathscr{B}(E)$ corresponds to a unique minimal, non-zero linear subspace of $E$, that is, a one-dimensional subspace, and therefore $\mathscr{L}$ is fixed. This conclusion is also an easy consequence of our work, as outlined in Remark 1.2(ii), below. By contrast, this statement is false whenever $E$ is infinite-dimensional because the two-sided ideal $\mathscr{F}(E)$ of finite-rank operators is proper, so that, by Krull's theorem, it is contained in a maximal left ideal, which cannot be fixed since, for each $x \in E \backslash\{0\}$, there is a finite-rank operator $T$ on $E$ such that $T x \neq 0$.

Inspired by these observations, the first-named author raised the following two questions for an infinite-dimensional Banach space $E$ :

(I) Does $\mathscr{B}(E)$ always contain a maximal left ideal which is not finitely generated?

(II) Is every finitely-generated, maximal left ideal of $\mathscr{B}(E)$ necessarily fixed?

In the light of the previous paragraph, we note that a positive answer to (II) would imply a positive answer to (I). Moreover, for similar reasons, it seems natural to consider also the following, formally more specific, variant of Question (II):

(III) Is the ideal $\mathscr{F}(E)$ of finite-rank operators ever contained in a finitely-generated, maximal left ideal of $\mathscr{B}(E)$ ?

The answers to the above questions depend only on the isomorphism class of the Banach space $E$. This follows from a theorem of Eidelheit, which states that two Banach spaces $E$ and $F$ are isomorphic if and only if the corresponding Banach algebras $\mathscr{B}(E)$ and $\mathscr{B}(F)$ are isomorphic (e.g., see [9, Theorem 2.5.7]).

After presenting some preliminary material in Section 2, we shall use a counting argument in Section 3 to answer Question (I) positively for a large class of Banach spaces, including all separable Banach spaces which contain an infinite-dimensional, closed, complemented subspace with an unconditional basis and, more generally, all separable Banach spaces with an unconditional Schauder decomposition (see Corollary 3.3 for details).

We then turn our attention to Questions (II) and (III). The main conclusion of Section 4 is that, for a given Banach space E, they are equivalent, in the sense that Question (II) has a positive answer if and only if Question (III) has a negative answer. This is an immediate consequence of the following dichotomy theorem for maximal left ideals, which can be viewed as the analogue of the fact that an ultrafilter on a set $M$ is either fixed (in the sense that it consists of precisely those subsets of $M$ which contain a fixed element $x \in M$ ), or it contains the Fréchet filter of all cofinite subsets of $M$.

Theorem 1.1 (Dichotomy for maximal left ideals). Let $E$ be a non-zero Banach space. Then, for each maximal left ideal $\mathscr{L}$ of $\mathscr{B}(E)$, exactly one of the following two alternatives holds:

(i) $\mathscr{L}$ is fixed; or

(ii) $\mathscr{L}$ contains $\mathscr{F}(E)$. 
Remark 1.2. (i) The result that we shall prove in Section 4 is in fact slightly stronger than Theorem 1.1, but also more technical to state, since it involves the larger ideal of inessential operators instead of $\mathscr{F}(E)$ (see Corollary 4.2 for details).

(ii) Let $E$ be a non-zero, finite-dimensional Banach space. Then $\mathscr{F}(E)=\mathscr{B}(E)$, so that no proper left ideal of $\mathscr{B}(E)$ satisfies condition (ii) of Theorem 1.1. Hence Theorem 1.1 implies that each maximal left ideal of $\mathscr{B}(E)$ is fixed.

We shall also show that a conclusion similar to that of Theorem 1.1 holds for closed left ideals of $\mathscr{B}(E)$ that are not necessarily maximal provided that either the underlying Banach space $E$ is reflexive, or that we restrict our attention to the closed left ideals which are finitely generated.

Theorem 1.3 (Dichotomy for closed left ideals). Let $E$ be a non-zero Banach space, let $\mathscr{L}$ be a closed left ideal of $\mathscr{B}(E)$, and suppose that either $E$ is reflexive or $\mathscr{L}$ is finitely generated (or both). Then exactly one of the following two alternatives holds:

(i) $\mathscr{L}$ is contained in a fixed maximal left ideal; or

(ii) $\mathscr{L}$ contains $\mathscr{F}(E)$.

We note that Theorems 1.1 and 1.3 are genuine dichotomies in the sense that in both theorems the two alternatives (i) and (ii) are mutually exclusive because, as observed above, no fixed maximal left ideal of $\mathscr{B}(E)$ contains $\mathscr{F}(E)$.

The purpose of Sections 5 and 6 is to show that Question (II) has a positive answer for many Banach spaces, both 'classical' and more 'exotic' ones. We can summarize our results as follows, and refer to Sections 5 and 6 for full details, including precise definitions of any unexplained terminology.

Theorem 1.4. Let $E$ be a Banach space which satisfies one of the following six conditions:

(i) E has a Schauder basis and is complemented in its bidual;

(ii) $E$ is isomorphic to the dual space of a Banach space with a Schauder basis;

(iii) $E$ is an injective Banach space;

(iv) $E=c_{0}(\Gamma), E=H$, or $E=c_{0}(\Gamma) \oplus H$, where $\Gamma$ is a non-empty index set and $H$ is a Hilbert space;

(v) E is a Banach space which has few operators;

(vi) $E=C(K)$, where $K$ is a compact Hausdorff space without isolated points, and each operator on $C(K)$ is a weak multiplication.

Then each finitely-generated, maximal left ideal of $\mathscr{B}(E)$ is fixed.

On the other hand, there is a Banach space for which the answer to Question (II) is negative; this is the main result of Section 7. Its statement involves Argyros-Haydon's Banach space having very few operators. We denote this space by $X_{\mathrm{AH}}$, and refer to Theorem 7.5 for a summary of its main properties.

Theorem 1.5. Let $E=X_{\mathrm{AH}} \oplus \ell_{\infty}$. Then the set

$$
\mathscr{K}_{1}=\left\{\left(\begin{array}{ll}
T_{1,1} & T_{1,2} \\
T_{2,1} & T_{2,2}
\end{array}\right) \in \mathscr{B}(E): T_{1,1} \text { is compact }\right\}
$$


is a maximal two-sided ideal of codimension one in $\mathscr{B}(E)$, and hence also a maximal left ideal. Moreover, $\mathscr{K}_{1}$ is singly generated as a left ideal, and it is not fixed.

This theorem suggests in particular that the Banach space $E=X_{\mathrm{AH}} \oplus \ell_{\infty}$ is a natural candidate for providing a negative answer to Question (I). However, as we shall also show in Section 7, it does not.

Theorem 1.6. Let $E=X_{\mathrm{AH}} \oplus \ell_{\infty}$. Then the ideal $\mathscr{K}_{1}$ given by (1.2) is the unique non-fixed, finitely-generated, maximal left ideal of $\mathscr{B}(E)$. Hence

$$
\mathscr{W}_{2}=\left\{\left(\begin{array}{ll}
T_{1,1} & T_{1,2} \\
T_{2,1} & T_{2,2}
\end{array}\right) \in \mathscr{B}(E): T_{2,2} \text { is weakly compact }\right\},
$$

which is a maximal two-sided ideal of $\mathscr{B}(E)$, is not contained in any finitely-generated, maximal left ideal of $\mathscr{B}(E)$.

To conclude this summary of our results, let us point out that Question (II) remains open in some important cases, notably for $E=C(K)$, where $K$ is any infinite, compact metric space such that $C(K) \approx c_{0}$.

As a final point, we shall explain how our work fits into a more general context. The main motivation behind Question (I) comes from the fact that it is the special case where $\mathscr{A}=\mathscr{B}(E)$ for a Banach space $E$ of the following conjecture, raised and discussed in [10]:

Let $\mathscr{A}$ be a unital Banach algebra such that every maximal left ideal of $\mathscr{A}$ is finitely generated. Then $\mathscr{A}$ is finite-dimensional.

A stronger form of this conjecture in the case where $\mathscr{A}$ is commutative was proved by Ferreira and Tomassini [13]; extensions of this result are given in [10]. The conjecture is also known to be true for $C^{*}$-algebras, although we have not been able to locate a precise statement of this result in the literature. We are very grateful to David Blecher and Mikael Rørdam for having communicated proofs of it to us. Blecher and the second author [6] are currently working on a generalization of this result to the class of Hilbert $C^{*}$-modules.

The conjecture is suggested by Sinclair and Tullo's theorem [42] which states that a Banach algebra $\mathscr{A}$ is finite dimensional if each closed left ideal of $\mathscr{A}$ (not just each maximal one) is finitely generated. This result has been generalized by Boudi [7], who showed that the conclusion that $\mathscr{A}$ is finite dimensional remains true under the formally weaker hypothesis that each closed left ideal of $\mathscr{A}$ is countably generated. Boudi's theorem can in fact be deduced from Sinclair and Tullo's theorem because a closed, countably-generated left ideal is necessarily finitely generated by [10, Proposition 1.5].

Another result that is related to our general theme, but of a different flavour from those just mentioned, is due to Grønbæk [20, Proposition 7.3], who has shown that, for a Banach space $E$ with the approximation property, the mapping

$$
F \mapsto \overline{\operatorname{span}}\{x \otimes \lambda: x \in E, \lambda \in F\}
$$

is an isomorphism of the lattice of closed linear subspaces $F$ of the dual space of $E$ onto the lattice of closed left ideals of the Banach algebra of compact operators on $E$; we refer to the next section for details of the notation and terminology used in this statement. 


\section{Preliminaries}

Our notation is mostly standard. We write $|\Gamma|$ for the cardinality of a set $\Gamma$. As usual, $\aleph_{0}$ and $\aleph_{1}$ denote the first and second infinite cardinals, respectively, while $\mathfrak{c}=2^{\aleph_{0}}$ is the cardinality of the continuum.

Let $E$ be a Banach space, always supposed to be over the complex field $\mathbb{C}$. We denote by $I_{E}$ the identity operator on $E$. For a non-empty set $\Gamma$, we define

$$
\ell_{\infty}(\Gamma, E)=\left\{f: \Gamma \rightarrow E:\|f\|_{\infty}<\infty\right\}, \quad \text { where } \quad\|f\|_{\infty}=\sup _{\gamma \in \Gamma}\|f(\gamma)\|,
$$

so that $\ell_{\infty}(\Gamma, E)$ is a Banach space with respect to the norm $\|\cdot\|_{\infty}$. The following special conventions apply:

- $\ell_{\infty}(\Gamma)=\ell_{\infty}(\Gamma, \mathbb{C})$;

- $\ell_{\infty}=\ell_{\infty}(\mathbb{N})$;

- $E^{n}=\ell_{\infty}(\{1, \ldots, n\}, E)$ for each $n \in \mathbb{N}$.

We write $E^{*}$ for the (continuous) dual space of the Banach space $E$. The duality bracket between $E$ and $E^{*}$ is $\langle\cdot, \cdot\rangle$, while $\kappa_{E}: E \rightarrow E^{* *}$ denotes the canonical embedding of $E$ into its bidual. By an operator, we understand a bounded, linear operator between Banach spaces; we write $\mathscr{B}(E, F)$ for the set of all operators from $E$ to another Banach space $F$, and denote by $T^{*} \in \mathscr{B}\left(F^{*}, E^{*}\right)$ the adjoint of an operator $T \in \mathscr{B}(E, F)$.

We shall require the following standard notions for $T \in \mathscr{B}(E, F)$ :

(i) $T$ is a finite-rank operator if it has finite-dimensional range. We write $\mathscr{F}(E, F)$ for the set of finite-rank operators from $E$ to $F$. It is well known that

$$
\mathscr{F}(E, F)=\operatorname{span}\left\{y \otimes \lambda: y \in F, \lambda \in E^{*}\right\},
$$

where $y \otimes \lambda$ denotes the rank-one operator given by

$$
y \otimes \lambda: x \mapsto\langle x, \lambda\rangle y, \quad E \rightarrow F \quad\left(y \in F, \lambda \in E^{*}\right) .
$$

The following elementary observation will be used several times:

$$
R(y \otimes \lambda) S=(R y) \otimes\left(S^{*} \lambda\right) \quad\left(S \in \mathscr{B}(D, E), y \in F, \lambda \in E^{*}, R \in \mathscr{B}(F, G)\right),
$$

valid for any Banach spaces $D, E, F$, and $G$.

(ii) $T$ is compact if the image under $T$ of the unit ball of $E$ is a relatively norm-compact subset of $F$. We write $\mathscr{K}(E, F)$ for the set of compact operators from $E$ to $F$.

(iii) $T$ is weakly compact if the image under $T$ of the unit ball of $E$ is a relatively weakly compact subset of $F$. We write $\mathscr{W}(E, F)$ for the set of weakly compact operators from $E$ to $F$.

(iv) $T$ is bounded below if, for some $\varepsilon>0$, we have $\|T x\| \geqslant \varepsilon\|x\|$ for each $x \in E$; or, equivalently, $T$ is injective and has closed range. This notion is dual to surjectivity in the following precise sense (e.g., see [34, Theorem 3.1.22]):

$T$ is surjective $\Longleftrightarrow T^{*}$ is bounded below, $T$ is bounded below $\Longleftrightarrow T^{*}$ is surjective. 
(v) $T$ is strictly singular if no restriction of $T$ to an infinite-dimensional subspace of $E$ is bounded below; that is, for each $\varepsilon>0$, each infinite-dimensional subspace of $E$ contains a unit vector $x$ such that $\|T x\| \leqslant \varepsilon$. We write $\mathscr{S}(E, F)$ for the set of strictly singular operators from $E$ to $F$.

(vi) $T$ is a Fredholm operator if both of the quantities

$$
\alpha(T)=\operatorname{dim} \operatorname{ker} T \quad \text { and } \quad \beta(T)=\operatorname{dim}(F / T(E))
$$

are finite, in which case $T$ has closed range, and its Fredholm index is defined by

$$
i(T)=\alpha(T)-\beta(T) \in \mathbb{Z} .
$$

We write $\Phi(E, F)$ for the set of Fredholm operators from $E$ to $F$.

(vii) $T$ is an upper semi-Fredholm operator if it has finite-dimensional kernel and closed range. We write $\Phi_{+}(E, F)$ for the set of upper semi-Fredholm operators from $E$ to $F$.

(viii) $T$ is inessential if $I_{E}-S T$ is a Fredholm operator for each $S \in \mathscr{B}(F, E)$. We write $\mathscr{E}(E, F)$ for the set of inessential operators from $E$ to $F$.

In line with common practice, we set $\mathscr{I}(E)=\mathscr{I}(E, E)$ whenever $\mathscr{I}$ denotes one of the eight classes $\mathscr{B}, \mathscr{F}, \mathscr{K}, \mathscr{W}, \mathscr{S}, \Phi, \Phi_{+}$, and $\mathscr{E}$ of operators introduced above. Of these classes, $\mathscr{B}, \mathscr{F}, \mathscr{K}, \mathscr{W}, \mathscr{S}$, and $\mathscr{E}$ define operator ideals in the sense of Pietsch, all of which except $\mathscr{F}$ are closed. The following inclusions hold in general:

$$
\mathscr{F}(E, F) \subseteq \mathscr{K}(E, F) \subseteq(\mathscr{W}(E, F) \cap \mathscr{S}(E, F)) \subseteq \mathscr{S}(E, F) \subseteq \mathscr{E}(E, F) \subseteq \mathscr{B}(E, F) .
$$

We remark that a left, right, or two-sided ideal of $\mathscr{B}(E)$ is proper if and only if it does not contain the identity operator $I_{E}$. This shows in particular that the two-sided ideal $\mathscr{E}(E)$ (and hence also $\mathscr{F}(E), \mathscr{K}(E)$, and $\mathscr{S}(E)$ ) is proper whenever $E$ is infinite-dimensional.

We shall require two perturbation results for (semi-)Fredholm operators. Firstly, the set of Fredholm operators is stable under inessential perturbations, and the Fredholm index is preserved under such perturbations (see [26, Theorem 6]):

$$
S+T \in \Phi(E, F) \quad \text { with } \quad i(S+T)=i(T) \quad(S \in \mathscr{E}(E, F), T \in \Phi(E, F)) .
$$

Secondly, the set of upper semi-Fredholm operators is stable under strictly singular perturbations (see [31, Proposition 2.c.10]):

$$
S+T \in \Phi_{+}(E, F) \quad\left(S \in \mathscr{S}(E, F), T \in \Phi_{+}(E, F)\right) .
$$

The following notion is central to this paper. Let $\Gamma$ be a non-empty subset of $\mathscr{B}(E)$ for some Banach space $E$. The left ideal generated by $\Gamma$ is the smallest left ideal $\mathscr{L}_{\Gamma}$ of $\mathscr{B}(E)$ that contains $\Gamma$. It can be described explicitly as

$$
\mathscr{L}_{\Gamma}=\left\{\sum_{j=1}^{n} S_{j} T_{j}: S_{1}, \ldots, S_{n} \in \mathscr{B}(E), T_{1}, \ldots, T_{n} \in \Gamma, n \in \mathbb{N}\right\} .
$$

A left ideal $\mathscr{L}$ of $\mathscr{B}(E)$ is singly (respectively, finitely, countably) generated if $\mathscr{L}=\mathscr{L}_{\Gamma}$ for some singleton (respectively, non-empty and finite, countable) subset $\Gamma$ of $\mathscr{B}(E)$. 
In the case where $\Gamma$ is non-empty and finite, say $\Gamma=\left\{T_{1}, \ldots, T_{n}\right\},(2.7)$ simplifies to

$$
\mathscr{L}_{\Gamma}=\left\{\sum_{j=1}^{n} S_{j} T_{j}: S_{1}, \ldots, S_{n} \in \mathscr{B}(E)\right\}=\left\{S \Psi_{\Gamma}: S \in \mathscr{B}\left(E^{n}, E\right)\right\},
$$

where we have introduced the operator

$$
\Psi_{\Gamma}=\sum_{j=1}^{n} \iota_{j} T_{j} \in \mathscr{B}\left(E, E^{n}\right) ;
$$

here $\iota_{j}: E \rightarrow E^{n}$ denotes the canonical $j^{\text {th }}$ coordinate embedding. (Note that, strictly speaking, the definition (2.9) of $\Psi_{\Gamma}$ requires that $\Gamma$ be an ordered $n$-tuple, not just a finite set. Whenever we consider $\Psi_{\Gamma}$, the ordering of $\Gamma$ is always understood to be by increasing index: $\Gamma=\left(T_{1}, \ldots, T_{n}\right)$.)

The operator $\Psi_{\Gamma}$, together with its counterpart for infinite $\Gamma$, which we shall introduce in Section 4, will play a key role in our work. We shall give here only one, very simple, application of $\Psi_{\Gamma}$, showing that each finitely-generated left ideal of operators is already singly generated for most 'classical' Banach spaces.

Proposition 2.1. Let $E$ be a Banach space which contains a complemented subspace that is isomorphic to $E \oplus E$. Then each finitely-generated left ideal of $\mathscr{B}(E)$ is singly generated.

Proof. Let $\Gamma$ be a non-empty, finite subset of $\mathscr{B}(E)$, and set $n=|\Gamma| \in \mathbb{N}$. By the assumption, $E$ contains a complemented subspace which is isomorphic to $E^{n}$, and hence $I_{E^{n}}=V U$ for some operators $U \in \mathscr{B}\left(E^{n}, E\right)$ and $V \in \mathscr{B}\left(E, E^{n}\right)$. We shall now complete the proof by showing that the left ideal $\mathscr{L}_{\Gamma}$ is generated by the single operator $T=U \Psi_{\Gamma} \in \mathscr{B}(E)$.

By (2.8), we have $T \in \mathscr{L}_{\Gamma}$, so that $\mathscr{L}_{\{T\}} \subseteq \mathscr{L}_{\Gamma}$.

Conversely, each operator $R \in \mathscr{L}_{\Gamma}$ has the form $R=S \Psi_{\Gamma}$ for some $S \in \mathscr{B}\left(E^{n}, E\right)$ by (2.8), and therefore $R=S(V U) \Psi_{\Gamma}=(S V) T \in \mathscr{L}_{\{T\}}$.

Our next result collects some basic facts about the fixed maximal left ideals of $\mathscr{B}(E)$, most of which were already stated in the Introduction.

Proposition 2.2. Let $x$ and $y$ be non-zero elements of a Banach space E. Then:

(i) the set $\mathscr{M} \mathscr{L}_{x}$ given by (1.1) is the left ideal of $\mathscr{B}(E)$ generated by the projection $I_{E}-x \otimes \lambda$, where $\lambda \in E^{*}$ is any functional such that $\langle x, \lambda\rangle=1$;

(ii) the left ideal $\mathscr{M} \mathscr{L}_{x}$ is maximal;

(iii) $\mathscr{M} \mathscr{L}_{x}=\mathscr{M} \mathscr{L}_{y}$ if and only if $x$ and $y$ are proportional.

In particular, $\mathscr{B}(E)$ contains $|E|$ distinct, fixed maximal left ideals whenever $E$ is infinitedimensional.

Proof. (i). Let $P=I_{E}-x \otimes \lambda$. The set $\mathscr{M} \mathscr{L}_{x}$ is clearly a left ideal which contains $P$, and hence $\mathscr{L}_{\{P\}} \subseteq \mathscr{M} \mathscr{L}_{x}$. The reverse inclusion holds because, by (2.2), $T(x \otimes \lambda)=0$ for each $T \in \mathscr{M} \mathscr{L}_{x}$, so that $T=T P \in \mathscr{L}_{\{P\}}$.

(ii). The left ideal $\mathscr{M} \mathscr{L}_{x}$ is evidently proper. To verify that it is maximal, suppose that $T \in \mathscr{B}(E) \backslash \mathscr{M} \mathscr{L}_{x}$. Then $T x \neq 0$, so that $\langle T x, \mu\rangle=1$ for some $\mu \in E^{*}$. The operator 
$S=I_{E}-(x \otimes \mu) T$ belongs to $\mathscr{M} \mathscr{L}_{x}$ because $(x \otimes \mu) T x=\langle T x, \mu\rangle x=x$, and consequently

$$
I_{E}=S+(x \otimes \mu) T \in \mathscr{M} \mathscr{L}_{x}+\mathscr{L}_{\{T\}} .
$$

(iii). It is clear that $\mathscr{M} \mathscr{L}_{x}=\mathscr{M} \mathscr{L}_{y}$ if $x$ and $y$ are proportional. We prove the converse by contraposition. Suppose that $x$ and $y$ are linearly independent. Then we can take $\lambda \in E^{*}$ such that $\langle x, \lambda\rangle=1$ and $\langle y, \lambda\rangle=0$, and hence $x \otimes \lambda \in \mathscr{M} \mathscr{L}_{y} \backslash \mathscr{M} \mathscr{L}_{x}$.

We conclude this preliminary section with the observation that the answer to the analogue of Question (I) for two-sided ideals is negative, as the following example shows.

Example 2.3. Consider the Hilbert space $H=\ell_{2}\left(\aleph_{1}\right)$, and take a projection $P \in \mathscr{B}(H)$ with separable, infinite-dimensional range. The ideal classification of Gramsch [17] and Luft [33] implies that the ideal $\mathscr{X}(H)$ of operators with separable range is the unique maximal two-sided ideal of $\mathscr{B}(H)$. Given $T \in \mathscr{X}(H)$, let $Q \in \mathscr{B}(H)$ be the orthogonal projection onto $\overline{T(H)}$. Then $T=Q T$, and also $Q=V P U$ for some operators $U, V \in \mathscr{B}(H)$, so that $T=V P U T$. Hence $\mathscr{X}(H)$ is the two-sided ideal of $\mathscr{B}(H)$ generated by the single operator $P$. Since $\mathscr{X}(H)$ is the only maximal two-sided ideal of $\mathscr{B}(H)$, we conclude that each maximal two-sided ideal of $\mathscr{B}(H)$ is singly generated, and therefore the analogue of Question (I) for two-sided ideals has a negative answer.

With slightly more work, we can give a similar example for a separable Banach space. To this end, consider the $p^{\text {th }}$ quasi-reflexive James space $J_{p}$ for some $p \in(1, \infty)$. Edelstein and Mityagin [12] observed that the two-sided ideal $\mathscr{W}\left(J_{p}\right)$ of weakly compact operators is maximal because it has codimension one in $\mathscr{B}\left(J_{p}\right)$. The fifth-named author [28, Theorem 4.16] has shown that $\mathscr{W}\left(J_{p}\right)$ is the unique maximal two-sided ideal of $\mathscr{B}\left(J_{p}\right)$. His work also implies that $\mathscr{W}\left(J_{p}\right)$ is singly generated as a two-sided ideal, as we shall now explain. Let

$$
J_{p}^{(\infty)}=\left(\bigoplus_{n \in \mathbb{N}} J_{p}^{(n)}\right)_{\ell_{p}}, \quad \text { where } \quad J_{p}^{(n)}=\left\{\left(\alpha_{j}\right)_{j \in \mathbb{N}} \in J_{p}: \alpha_{j}=0(j>n)\right\} \quad(n \in \mathbb{N}) .
$$

This is clearly a reflexive Banach space, which is isomorphic to a complemented subspace of $J_{p}$. (The latter observation is due to Edelstein and Mityagin [12, Lemma 6(d)]. An alternative approach can be found in [28, Proposition $4.4(\mathrm{iv})]$.) Take a projection $P \in \mathscr{B}\left(J_{p}\right)$ whose range is isomorphic to $J_{p}^{(\infty)}$. By [29, Theorem 4.3], we have

$$
\mathscr{W}\left(J_{p}\right)=\left\{T S: S \in \mathscr{B}\left(J_{p}, J_{p}^{(\infty)}\right), T \in \mathscr{B}\left(J_{p}^{(\infty)}, J_{p}\right)\right\}=\left\{V P U: U, V \in \mathscr{B}\left(J_{p}\right)\right\},
$$

so that $\mathscr{W}\left(J_{p}\right)$ is the two-sided ideal of $\mathscr{B}\left(J_{p}\right)$ generated by the single operator $P$.

On the other hand, Corollary 4.8 , below, will show that $\mathscr{W}\left(J_{p}\right)$ is not finitely generated as a left ideal because $J_{p}$ is non-reflexive.

\section{Counting maximal Left iDeals}

Let $E$ be an infinite-dimensional Banach space. An infinite family $\left(E_{\gamma}\right)_{\gamma \in \Gamma}$ of non-zero, closed subspaces of $E$ is an unconditional Schauder decomposition of $E$ if, for each $x \in E$, there is a unique family $\left(x_{\gamma}\right)_{\gamma \in \Gamma}$ with $x_{\gamma} \in E_{\gamma}$ for each $\gamma \in \Gamma$ such that the series $\sum_{\gamma \in \Gamma} x_{\gamma}$ 
converges unconditionally to $x$. In this case we can associate a projection $P_{\Upsilon} \in \mathscr{B}(E)$ with each subset $\Upsilon$ of $\Gamma$ by the definitions

$$
P_{\emptyset}=0 \quad \text { and } \quad P_{\Upsilon} x=\sum_{\gamma \in \Upsilon} x_{\gamma} \quad(x \in E) \quad \text { for } \quad \Upsilon \neq \emptyset,
$$

where $\left(x_{\gamma}\right)_{\gamma \in \Gamma}$ is related to $x$ as above.

Using this notion, we can transfer a classical algebraic result of Rosenberg $[40]$ to $\mathscr{B}(E)$.

Proposition 3.1. Let $E$ be a Banach space with an unconditional Schauder decomposition $\left(E_{\gamma}\right)_{\gamma \in \Gamma}$. Then the Banach algebra $\mathscr{B}(E)$ contains at least $2^{2^{|\Gamma|}}$ maximal left ideals which are not fixed.

Proof. The power set $\mathfrak{P}(\Gamma)$ of $\Gamma$ is a Boolean algebra, and $\mathfrak{I}=\{\Upsilon \in \mathfrak{P}(\Gamma):|\Upsilon|<|\Gamma|\}$ is a proper Boolean ideal of $\mathfrak{P}(\Gamma)$. Since $\Gamma$ is infinite, a classical result of Pospíšil (see [38], or $[8$, Corollary 7.4$]$ for an exposition) states that the collection $\mathbb{M}_{\mathfrak{I}}$ of maximal Boolean ideals of $\mathfrak{P}(\Gamma)$ containing $\mathfrak{I}$ has cardinality $2^{2^{|\Gamma|}}$.

For each $\mathfrak{M} \in \mathbb{M}_{\mathfrak{I}}$, let $\mathrm{P}(\mathfrak{M})=\left\{P_{\Upsilon}: \Upsilon \in \mathfrak{M}\right\} \subseteq \mathscr{B}(E)$, where $P_{\Upsilon}$ is the projection given by (3.1). Assume towards a contradiction that the left ideal $\mathscr{L}_{\mathrm{P}(\mathfrak{M})}$ is not proper. Then, for some $n \in \mathbb{N}$, there are operators $T_{1}, \ldots, T_{n} \in \mathscr{B}(E)$ and sets $\Upsilon_{1}, \ldots, \Upsilon_{n} \in \mathfrak{M}$ such that $I_{E}=\sum_{j=1}^{n} T_{j} P_{\Upsilon_{j}}$. Right-composing both sides of this identity with the projection $P_{\Gamma \backslash \Upsilon}$, where $\Upsilon=\bigcup_{j=1}^{n} \Upsilon_{j} \in \mathfrak{M}$, we obtain $P_{\Gamma \backslash \Upsilon}=0$, so that $\Gamma=\Upsilon \in \mathfrak{M}$, which contradicts the fact that $\mathfrak{M}$ is a proper Boolean ideal.

We can therefore choose a maximal left ideal $\mathscr{M}_{\mathfrak{M}}$ of $\mathscr{B}(E)$ such that $\mathscr{L}_{\mathrm{P}(\mathfrak{M})} \subseteq \mathscr{M}_{\mathfrak{M}}$. This maximal left ideal $\mathscr{M}_{\mathfrak{M}}$ cannot be fixed because, for each $x \in E \backslash\{0\}$, we have $x=\sum_{\gamma \in \Gamma} P_{\{\gamma\}} x$, so that $P_{\{\gamma\}} x \neq 0$ for some $\gamma \in \Gamma$. Hence $P_{\{\gamma\}} \notin \mathscr{M} \mathscr{L}_{x}$, but on the other hand $P_{\{\gamma\}} \in \mathscr{L}_{\mathrm{P}(\mathfrak{M})} \subseteq \mathscr{M}_{\mathfrak{M}}$ since $\{\gamma\} \in \mathfrak{I} \subseteq \mathfrak{M}$.

Consequently we have a mapping $\mathfrak{M} \mapsto \mathscr{M}_{\mathfrak{M}}$ from $\mathbb{M}_{\mathfrak{I}}$ into the set of non-fixed, maximal left ideals of $\mathscr{B}(E)$. We shall complete the proof by showing that this mapping is injective. Suppose that $\mathfrak{M}, \mathfrak{N} \in \mathbb{M}_{\mathfrak{I}}$ are distinct, and take a set $\Upsilon \in \mathfrak{M} \backslash \mathfrak{N}$. The maximality of $\mathfrak{N}$ implies that $\Gamma \backslash \Upsilon \in \mathfrak{N}$, and therefore

$$
I_{E}=P_{\Upsilon}+P_{\Gamma \backslash \Upsilon} \in \mathscr{L}_{\mathrm{P}(\mathfrak{M})}+\mathscr{L}_{\mathrm{P}(\mathfrak{N})} \subseteq \mathscr{M}_{\mathfrak{M}}+\mathscr{M}_{\mathfrak{N}}
$$

Thus, since the left ideals $\mathscr{M}_{\mathfrak{M}}$ and $\mathscr{M}_{\mathfrak{N}}$ are proper, they are distinct.

Corollary 3.2. Let $E$ be a Banach space with an unconditional Schauder decomposition $\left(E_{\gamma}\right)_{\gamma \in \Gamma}$, and suppose that $E$ contains a dense subset $D$ such that $2^{|D|}<2^{2^{|\Gamma|}}$. Then $\mathscr{B}(E)$ contains at least $2^{2^{|\Gamma|}}$ maximal left ideals which are not finitely generated.

Proof. Since each point of $E$ is the limit point of a sequence in $D$, we have $|E| \leqslant|D|^{\aleph_{0}}$. Further, each operator on $E$ is uniquely determined by its action on $D$, and consequently

$$
|\mathscr{B}(E)| \leqslant\left|E^{D}\right|=|E|^{|D|} \leqslant\left(|D|^{\aleph_{0}}\right)^{|D|}=|D|^{|D|}=2^{|D|},
$$

where the final equality follows from a standard result (e.g., see [22, Lemma 5.6]). Hence $\mathscr{B}(E)$ contains at most $\left(2^{|D|}\right)^{\aleph_{0}}=2^{|D|}$ countable subsets, so that $\mathscr{B}(E)$ contains at most 
$2^{|D|}$ countably-generated left ideals. On the other hand, Proposition 3.1 implies that there are at least $2^{2^{|\Gamma|}}$ distinct maximal left ideals of $\mathscr{B}(E)$. We have $2^{|D|}<2^{2^{|\Gamma|}}$ by the assumption, and hence $\mathscr{B}(E)$ contains at least $2^{2^{|\Gamma|}}$ maximal left ideals which are not countably generated, and thus not finitely generated.

The most important case of this corollary is as follows.

Corollary 3.3. Let $E$ be a separable Banach space with an unconditional Schauder decomposition $\left(E_{\gamma}\right)_{\gamma \in \Gamma}$. Then $\mathscr{B}(E)$ contains $2^{\mathfrak{c}}$ maximal left ideals which are not finitely generated.

Proof. The index set $\Gamma$ is necessarily countable because $E$ is separable. Hence, by Corollary $3.2, \mathscr{B}(E)$ contains at least $2^{\mathfrak{c}}$ maximal left ideals which are not finitely generated. On the other hand, (3.2) implies that $\mathscr{B}(E)$ has cardinality $\mathfrak{c}$, so that $\mathscr{B}(E)$ contains no more than $2^{\mathfrak{c}}$ distinct subsets.

Example 3.4. (i) Let $E$ be a Banach space which has an unconditional Schauder basis $\left(e_{n}\right)_{n \in \mathbb{N}}$. Then $E$ satisfies the conditions of Corollary 3.3, and hence $\mathscr{B}(E)$ contains $2^{\mathfrak{c}}$ maximal left ideals which are not finitely generated.

The class of Banach spaces which have an unconditional Schauder basis is large and includes for instance the classical sequence spaces $c_{0}$ and $\ell_{p}$ for $p \in[1, \infty)$, the Lebesgue spaces $L_{p}[0,1]$ for $p \in(1, \infty)$, the Lorentz and Orlicz sequence spaces $d_{w, p}$ and $h_{M}$ (e.g., see [31, Chapter 4]), the Tsirelson space $T$ (e.g., see [31, Example 2.e.1]), and the Schlumprecht space $S$ (see [41, Proposition 2]).

(ii) Suppose that $E$ is a Banach space which contains an infinite-dimensional, closed, complemented subspace $F$ with an unconditional Schauder decomposition $\left(F_{\gamma}\right)_{\gamma \in \Gamma}$. Then $E$ also has an unconditional Schauder decomposition, obtained by adding any closed, complementary subspace of $F$ to the collection $\left(F_{\gamma}\right)_{\gamma \in \Gamma}$.

In particular, generalizing (i), we see that each separable Banach space $E$ which contains an infinite-dimensional, closed, complemented subspace with an unconditional Schauder basis satisfies the conditions of Corollary 3.3, and hence $\mathscr{B}(E)$ contains $2^{\mathfrak{c}}$ maximal left ideals that are not finitely generated. This applies for instance to $E=L_{1}[0,1]$ because it contains a complemented copy of $\ell_{1}$ (e.g., see [1, Lemma 5.1.1]); to $E=C(K)$ for any infinite, compact metric space $K$ because $E$ contains a complemented copy of $c_{0}$ (e.g., see [1, Proposition 4.3.11]); to $E=J_{p}$ for $p \in(1, \infty)$, the $p^{\text {th }}$ quasi-reflexive James space, because $J_{p}$ contains a complemented copy of $\ell_{p}$ (see [12, Lemma $\left.6(\mathrm{~d})\right]$ or [28, Proposition 4.4(iii)]); and to $E=\mathscr{K}(X)$, where $X$ is any Banach space with an unconditional Schauder basis, because $E$ contains a complemented copy of $c_{0}$ consisting of the compact operators whose matrix representation with respect to the unconditional Schauder basis is diagonal.

(iii) There are separable Banach spaces $E$ such that $E$ has a unconditional Schauder decomposition $\left(E_{n}\right)_{n \in \mathbb{N}}$ with each $E_{n}$ finite-dimensional, but $E$ does not have an unconditional Schauder basis, notably Kalton and Peck's twisted $\ell_{p}$-spaces $Z_{p}$ for $p \in(1, \infty)$ (see [25, Corollary 9] and the remark following it). Each such Banach 
space $E$ satisfies the conditions of Corollary 3.3 , and hence $\mathscr{B}(E)$ contains $2^{\mathfrak{c}}$ maximal left ideals which are not finitely generated.

Remark 3.5. Corollary 3.3 is not true for all separable, infinite-dimensional Banach spaces. Indeed, we shall show in Theorem 6.2, below, that there are separable, infinitedimensional Banach spaces $E$ such that $\mathscr{B}(E)$ contains just one maximal left ideal which is not fixed, and this ideal is not finitely generated.

\section{Proofs of the Dichotomy Theorems 1.1 And 1.3}

The main purpose of this section is to prove Theorems 1.1 and 1.3.

Theorem 4.1. Let $E$ be a non-zero Banach space, and let $\mathscr{L}$ be a proper left ideal of $\mathscr{B}(E)$ such that $\mathscr{L}$ is not contained in any fixed maximal left ideal. Then the left ideal $\mathscr{L}+\mathscr{E}(E)$ is proper.

Proof. Suppose contrapositively that $\mathscr{L}$ is a left ideal of $\mathscr{B}(E)$ such that $\mathscr{L}+\mathscr{E}(E)=\mathscr{B}(E)$ and $\mathscr{L} \nsubseteq \mathscr{M} \mathscr{L}_{x}$ for each $x \in E \backslash\{0\}$. We shall then prove that $\mathscr{L}=\mathscr{B}(E)$. By the first assumption, we can write $I_{E}=R+S$ for some $R \in \mathscr{L}$ and $S \in \mathscr{E}(E)$, and hence $R=I_{E}-S$ is a Fredholm operator of index zero by (2.5). The set

$$
\mathscr{Z}=\{T \in \mathscr{L} \cap \Phi(E): i(T)=0\}
$$

is therefore non-empty.

Our next aim is to show that, for each $T \in \mathscr{Z}$ with $\alpha(T)>0$, there exists $U \in \mathscr{Z}$ such that $\alpha(U)=\alpha(T)-1$, where $\alpha(\cdot)$ is defined by (2.4). Indeed, take $x \in \operatorname{ker} T \backslash\{0\}$ and $y \in E \backslash T(E)$. Since $\mathscr{L} \nsubseteq \mathscr{M} \mathscr{L}_{x}$, we can find an operator $V \in \mathscr{L}$ such that $V x \neq 0$, and hence $\langle V x, \lambda\rangle=1$ for some $\lambda \in E^{*}$. Let $U=T+(y \otimes \lambda) V$. Then $U \in \mathscr{Z}$ because $T \in \mathscr{Z}$ and $(y \otimes \lambda) V \in \mathscr{L} \cap \mathscr{F}(E)$, and also $\alpha(U)=\alpha(T)-1$ because $\operatorname{ker} T=\operatorname{ker} U \oplus \mathbb{C} x$, as is easily verified.

The result established in the previous paragraph implies in particular that $\mathscr{Z}$ contains an operator $U$ with $\alpha(U)=0$. Hence $\beta(U)=\alpha(U)-i(U)=0$, so that $U$ is invertible, and consequently $\mathscr{L}=\mathscr{B}(E)$, as required.

This result has as an immediate consequence the following dichotomy, which generalizes Theorem 1.1 slightly because $\mathscr{F}(E) \subsetneq \mathscr{E}(E)$.

Corollary 4.2 (Strong dichotomy for maximal left ideals). Let $E$ be a non-zero Banach space. Then, for each maximal left ideal $\mathscr{L}$ of $\mathscr{B}(E)$, exactly one of the following two alternatives holds:

(i) $\mathscr{L}$ is fixed; or

(ii) $\mathscr{L}$ contains $\mathscr{E}(E)$.

Proof. Suppose that the maximal left ideal $\mathscr{L}$ of $\mathscr{B}(E)$ is not fixed. Then $\mathscr{L} \nsubseteq \mathscr{M} \mathscr{L}_{x}$ for each $x \in E \backslash\{0\}$, so that Theorem 4.1 implies that $\mathscr{L}+\mathscr{E}(E)$ is a proper left ideal of $\mathscr{B}(E)$. Hence, by the maximality of $\mathscr{L}$, we have $\mathscr{L}=\mathscr{L}+\mathscr{E}(E)$; that is, (ii) holds. 
Remark 4.3. Corollary 4.2 can be seen as a counterpart for maximal left ideals of the following observation due to the fifth-named author [28, Proposition 6.6]. Let $E$ be an infinite-dimensional Banach space. Then $\mathscr{E}(E)$ is contained in each maximal two-sided ideal of $\mathscr{B}(E)$.

Remark 4.4. Let $\mathscr{A}$ be a unital $C^{*}$-algebra. We write $a \mapsto a^{\star}$ for the involution on $\mathscr{A}$. (This should not be confused with the notation $T^{*}$ for the adjoint of an operator $T$ between Banach spaces used elsewhere in this paper.) A state on $\mathscr{A}$ is a norm-one functional $\lambda$ on $\mathscr{A}$ which is positive, in the sense that $\left\langle a^{\star} a, \lambda\right\rangle \geqslant 0$ for each $a \in \mathscr{A}$. Given a state $\lambda$ on $\mathscr{A}$, the set

$$
\mathscr{N}_{\lambda}=\left\{a \in \mathscr{A}:\left\langle a^{\star} a, \lambda\right\rangle=0\right\}
$$

is a closed left ideal of $\mathscr{A}$ by the Cauchy-Schwarz inequality (e.g., see [23, Proposition 4.5.1] or [35, p. 93]). The collection of all states on $\mathscr{A}$ forms a weak ${ }^{*}$-compact, convex subset of the dual space of $\mathscr{A}$, called the state space of $\mathscr{A}$. Its extreme points are the pure states on $\mathscr{A}$. Prosser [39, Theorem 6.2] has shown that the map $\lambda \mapsto \mathscr{N}_{\lambda}$ gives a bijective correspondence between the pure states on $\mathscr{A}$ and the maximal left ideals of $\mathscr{A}$; expositions of this result can be found in [24, Theorem 10.2.10] and [35, Theorem 5.3.5].

In the case where $\mathscr{A}=\mathscr{B}(H)$ for some Hilbert space $H$, the fixed maximal left ideals correspond to the vector states, which are defined as follows. Let $x \in H$ be a unit vector. Then the functional $\omega_{x}$ given by

$$
\left\langle T, \omega_{x}\right\rangle=(T x \mid x) \quad(T \in \mathscr{B}(H)),
$$

where $(\cdot \mid \cdot)$ denotes the inner product on $H$, is a pure state on $\mathscr{B}(H)$, called the vector state induced by $x$; and we have $\mathscr{M} \mathscr{L}_{x}=\mathscr{N}_{\omega_{x}}$, as is easy to check. The conclusion of Corollary 4.2 is known in this case because $\mathscr{K}(H)=\mathscr{E}(H)$, and by [24, Corollary 10.4.4] each pure state $\lambda$ on $\mathscr{B}(H)$ is either a vector state, or $\mathscr{K}(H) \subseteq$ ker $\lambda$, in which case $\mathscr{K}(H) \subseteq \mathscr{N}_{\lambda}$.

Finally, suppose that the Hilbert space $H$ is separable and infinite-dimensional. Then clearly $\mathscr{B}(H)$ has $\mathfrak{c}$ vector states, whereas it has $2^{\mathfrak{c}}$ pure states by [24, Proposition 10.4.15]. These conclusions also follow from Proposition 2.2 and Example 3.4(i), respectively.

We shall now turn our attention to the proof of Theorem 1.3. This requires some preparation. Let $E$ be a Banach space. For each non-empty, bounded subset $\Gamma$ of $\mathscr{B}(E)$, we can define an operator $\Psi_{\Gamma}: E \rightarrow \ell_{\infty}(\Gamma, E)$ by

$$
\left(\Psi_{\Gamma} x\right)(T)=T x \quad(x \in E, T \in \Gamma) .
$$

Note that, after natural identifications, this definition generalizes (2.9). Further, we can define a linear isometry $\Xi_{\Gamma}$ from the Banach space

$$
\ell_{1}\left(\Gamma, E^{*}\right)=\left\{g: \Gamma \rightarrow E^{*}: \sum_{T \in \Gamma}\|g(T)\|<\infty\right\}
$$

into $\ell_{\infty}(\Gamma, E)^{*}$ by

$$
\left\langle f, \Xi_{\Gamma} g\right\rangle=\sum_{T \in \Gamma}\langle f(T), g(T)\rangle \quad\left(f \in \ell_{\infty}(\Gamma, E), g \in \ell_{1}\left(\Gamma, E^{*}\right)\right) .
$$


Letting $\Omega_{\Gamma}=\Psi_{\Gamma}^{*} \Xi_{\Gamma} \in \mathscr{B}\left(\ell_{1}\left(\Gamma, E^{*}\right), E^{*}\right)$, we then obtain a commutative diagram

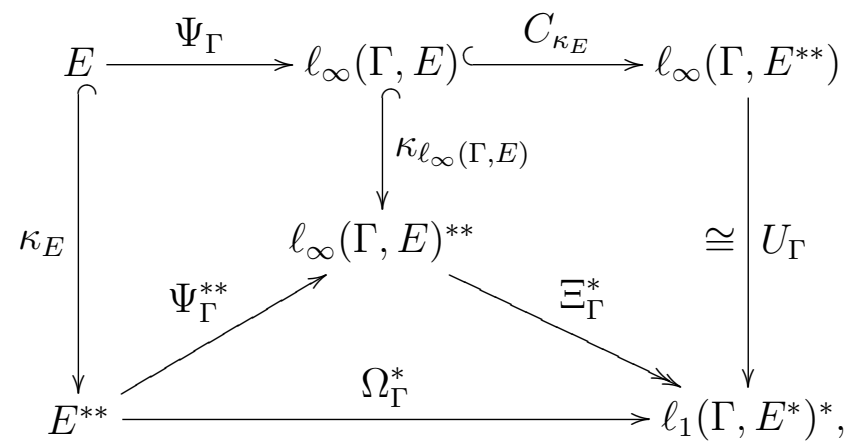

where $C_{\kappa_{E}}: f \mapsto \kappa_{E} \circ f$ is the composition operator induced by $\kappa_{E}$, while $U_{\Gamma}$ is the usual isometric identification of $\ell_{\infty}\left(\Gamma, E^{* *}\right)$ with the dual space of $\ell_{1}\left(\Gamma, E^{*}\right)$ given by

$$
\left\langle g, U_{\Gamma} f\right\rangle=\sum_{T \in \Gamma}\langle g(T), f(T)\rangle \quad\left(f \in \ell_{\infty}\left(\Gamma, E^{* *}\right), g \in \ell_{1}\left(\Gamma, E^{*}\right)\right) .
$$

The first of the following two lemmas explains the relevance of the operator $\Psi_{\Gamma}$ for our present purpose, while part (iii) of the second identifies the role of $\Omega_{\Gamma}$.

Lemma 4.5. Let $x$ be a non-zero element of a Banach space $E$, and let $\Gamma$ be a non-empty, bounded subset of $\mathscr{B}(E)$. Then $x \in \operatorname{ker} \Psi_{\Gamma}$ if and only if $\mathscr{L}_{\Gamma} \subseteq \mathscr{M} \mathscr{L}_{x}$.

Proof. By the definition (4.2) of $\Psi_{\Gamma}$, we have

$$
x \in \operatorname{ker} \Psi_{\Gamma} \Longleftrightarrow(T x=0 \quad(T \in \Gamma)) \quad \Longleftrightarrow \Gamma \subseteq \mathscr{M} \mathscr{L}_{x} \Longleftrightarrow \mathscr{L}_{\Gamma} \subseteq \mathscr{M} \mathscr{L}_{x},
$$

which gives the result.

Lemma 4.6. Let $E$ be a non-zero Banach space, and let $\Gamma$ be a non-empty, bounded subset of $\mathscr{B}(E)$ for which the corresponding left ideal $\mathscr{L}_{\Gamma}$ is closed.

(i) For each $\lambda \in E^{*}$, the set

$$
\mathscr{J}_{\lambda}=\{y \otimes \lambda: y \in E\}
$$

is a left ideal of $\mathscr{B}(E)$, and the following three conditions are equivalent:
(a) $\mathscr{J}_{\lambda} \subseteq \mathscr{L}_{\Gamma}$;
(b) $y \otimes \lambda \in \mathscr{L}_{\Gamma}$ for some $y \in E \backslash\{0\}$;
(c) $\lambda \in \Omega_{\Gamma}\left(\ell_{1}\left(\Gamma, E^{*}\right)\right)$.

(ii) The operator $\Omega_{\Gamma}$ has closed range.

(iii) The operator $\Omega_{\Gamma}$ is surjective if and only if $\mathscr{F}(E) \subseteq \mathscr{L}_{\Gamma}$.

Proof. (i). Equation (2.2) shows that $\mathscr{J}_{\lambda}$ is a left ideal.

The implication $(\mathrm{a}) \Rightarrow(\mathrm{b})$ is evident.

(b) $\Rightarrow\left(\right.$ c). Suppose that $y \otimes \lambda \in \mathscr{L}_{\Gamma}$ for some $y \in E \backslash\{0\}$, so that $y \otimes \lambda=\sum_{j=1}^{n} S_{j} T_{j}$ for some $n \in \mathbb{N}, S_{1}, \ldots, S_{n} \in \mathscr{B}(E)$ and $T_{1}, \ldots, T_{n} \in \Gamma$. We may suppose that $T_{1}, \ldots, T_{n}$ are 
distinct. Choose $\mu \in E^{*}$ such that $\langle y, \mu\rangle=1$, and define $g: \Gamma \rightarrow E^{*}$ by

$$
g(T)= \begin{cases}S_{j}^{*} \mu & \text { if } T=T_{j} \text { for some } j \in\{1, \ldots, n\}, \\ 0 & \text { otherwise. }\end{cases}
$$

Then $g$ has finite support, so that trivially it belongs to $\ell_{1}\left(\Gamma, E^{*}\right)$, and $\Omega_{\Gamma} g=\lambda$ because

$$
\begin{aligned}
\left\langle x, \Omega_{\Gamma} g\right\rangle=\left\langle\Psi_{\Gamma} x, \Xi_{\Gamma} g\right\rangle & =\sum_{T \in \Gamma}\langle T x, g(T)\rangle=\sum_{j=1}^{n}\left\langle T_{j} x, S_{j}^{*} \mu\right\rangle=\left\langle\sum_{j=1}^{n} S_{j} T_{j} x, \mu\right\rangle \\
& =\langle(y \otimes \lambda) x, \mu\rangle=\langle x, \lambda\rangle\langle y, \mu\rangle=\langle x, \lambda\rangle \quad(x \in E) .
\end{aligned}
$$

Hence $\lambda \in \Omega_{\Gamma}\left(\ell_{1}\left(\Gamma, E^{*}\right)\right)$.

(c) $\Rightarrow$ (a). Suppose that $\lambda=\Omega_{\Gamma} g$ for some $g \in \ell_{1}\left(\Gamma, E^{*}\right)$. Then, for each $y \in E$, we have

$$
(y \otimes \lambda) x=\left\langle x, \Omega_{\Gamma} g\right\rangle y=\sum_{T \in \Gamma}\langle T x, g(T)\rangle y=\sum_{T \in \Gamma}(y \otimes g(T)) T x \quad(x \in E) .
$$

The series $\sum_{T \in \Gamma}(y \otimes g(T)) T$ converges absolutely because $g \in \ell_{1}\left(\Gamma, E^{*}\right)$ and $\Gamma$ is bounded, and each term of this series belongs to the left ideal $\mathscr{L}_{\Gamma}$, which is closed by hypothesis. Hence the sum of the series, which is equal to $y \otimes \lambda$ by (4.5), also belongs to $\mathscr{L}_{\Gamma}$, and so $\mathscr{J}_{\lambda} \subseteq \mathscr{L}_{\Gamma}$

(ii). Suppose that $\left(\lambda_{j}\right)_{j \in \mathbb{N}}$ is a sequence in $\Omega_{\Gamma}\left(\ell_{1}\left(\Gamma, E^{*}\right)\right)$ which converges to $\lambda \in E^{*}$. By (i), we have $\mathscr{J}_{\lambda_{j}} \subseteq \mathscr{L}_{\Gamma}$ for each $j \in \mathbb{N}$. Hence

$$
y \otimes \lambda=\lim _{j \rightarrow \infty} y \otimes \lambda_{j} \in \mathscr{L}_{\Gamma} \quad(y \in E)
$$

because $\mathscr{L}_{\Gamma}$ is closed, so that $\lambda \in \Omega_{\Gamma}\left(\ell_{1}\left(\Gamma, E^{*}\right)\right)$ by another application of (i).

(iii). Suppose that $\Omega_{\Gamma}$ is surjective. Then (i) implies that $\mathscr{J}_{\lambda} \subseteq \mathscr{L}_{\Gamma}$ for each $\lambda \in E^{*}$, and consequently $\mathscr{F}(E) \subseteq \mathscr{L}_{\Gamma}$ by $(2.1)$.

Conversely, suppose that $\mathscr{F}(E) \subseteq \mathscr{L}_{\Gamma}$, and let $\lambda \in E^{*}$ be given. Since $\mathscr{J}_{\lambda} \subseteq \mathscr{F}(E)$, (i) implies that $\lambda \in \Omega_{\Gamma}\left(\ell_{1}\left(\Gamma, E^{*}\right)\right)$, so that $\Omega_{\Gamma}$ is surjective.

We can now characterize the closed left ideals of $\mathscr{B}(E)$ that contain $\mathscr{F}(E)$ as follows, provided either that $E$ is reflexive or that we restrict our attention to the closed left ideals that are finitely generated. Note that Theorem 1.3 is simply a restatement of the equivalence of conditions (a) and (e).

Theorem 4.7. Let $E$ be a non-zero Banach space, let $\mathscr{L}$ be a closed left ideal of $\mathscr{B}(E)$, and take a non-empty, bounded subset $\Gamma$ of $\mathscr{B}(E)$ such that $\mathscr{L}=\mathscr{L}_{\Gamma}$. Suppose that either $E$ is reflexive or $\Gamma$ is finite. Then the following five conditions are equivalent:

(a) no fixed maximal left ideal of $\mathscr{B}(E)$ contains $\mathscr{L}$;

(b) the operator $\Psi_{\Gamma}$ is injective;

(c) the operator $\Psi_{\Gamma}$ is bounded below;

(d) the operator $\Omega_{\Gamma}$ is surjective;

(e) $\mathscr{L}$ contains $\mathscr{F}(E)$. 
Proof. The following implications hold without any further assumptions on $E$ or $\Gamma$ :

Conditions (a) and (b) are equivalent by Lemma 4.5.

Conditions (d) and (e) are equivalent by Lemma 4.6(iii).

Evidently (c) implies (b).

Condition (d) implies (c). Indeed, suppose that $\Omega_{\Gamma}=\Psi_{\Gamma}^{*} \Xi_{\Gamma}$ is surjective. Then $\Psi_{\Gamma}^{*}$ is also surjective, and therefore $\Psi_{\Gamma}$ is bounded below by $(2.3)$.

The remaining implication, that (b) implies $(\mathrm{d})$, does require further assumptions. We consider first the case where $E$ is reflexive. The operators $\kappa_{E}$ and $C_{\kappa_{E}}$ in the diagram (4.4) are then isomorphisms, and so $\Psi_{\Gamma}$ and $\Omega_{\Gamma}^{*}$ are equal up to isomorphic identifications. Hence (2.3) implies that (c) and (d) are equivalent. Moreover, $\Omega_{\Gamma}$ has closed range by Lemma 4.6(ii). The same is therefore true for $\Psi_{\Gamma}$ by the closed range theorem (e.g., see [34, Theorem 3.1.21]), and so conditions (b) and (c) are also equivalent.

Secondly, suppose that the set $\Gamma$ is finite. Then the operator $\Xi_{\Gamma}: \ell_{1}\left(\Gamma, E^{*}\right) \rightarrow \ell_{\infty}(\Gamma, E)^{*}$ given by (4.3) is an isomorphism, and so $\Omega_{\Gamma}$ and $\Psi_{\Gamma}^{*}$ are equal up to isomorphic identifications. Hence the conclusion follows as in the first case.

As an easy consequence of this result, we obtain that the ideal of weakly compact operators is finitely generated as a left ideal only in the trivial case where it is not proper.

Corollary 4.8. The following three conditions are equivalent for a Banach space E:

(a) $\mathscr{W}(E)$ is finitely generated as a left ideal;

(b) $\mathscr{W}(E)=\mathscr{B}(E)$;

(c) the Banach space $E$ is reflexive.

Proof. The equivalence of (b) and (c) is standard, and (b) obviously implies (a).

To see that (a) implies (c), suppose that $\mathscr{W}(E)=\mathscr{L}_{\Gamma}$ for some non-empty, finite subset $\Gamma=\left\{T_{1}, \ldots, T_{n}\right\}$ of $\mathscr{B}(E)$. It clearly suffices to consider the case where $E$ is non-zero. Theorem 4.7 implies that the operator $\Psi_{\Gamma}$ is bounded below because $\mathscr{W}(E)$ contains $\mathscr{F}(E)$. Since the operators $T_{1}, \ldots, T_{n}$ are weakly compact, the same is true for $\Psi_{\Gamma}$ by the definition (2.9). Hence the Davis-Figiel-Johnson-Pełczyński factorization theorem (see [11], or [32, Theorem 2.g.11] for an exposition) implies that, for some reflexive Banach space $F$, there are operators $R \in \mathscr{B}(E, F)$ and $S \in \mathscr{B}\left(F, E^{n}\right)$ such that $\Psi_{\Gamma}=S R$. Now $R$ is bounded below because $\Psi_{\Gamma}$ is, and therefore $E$ is isomorphic to the subspace $R(E)$ of the reflexive space $F$, so that $E$ is reflexive.

We conclude this section with an example that shows that Theorem 4.7 may not be true if we drop the assumption that either the Banach space $E$ is reflexive or the set $\Gamma$ is finite. This requires the following easy variant of Lemma 4.6(i).

Lemma 4.9. Let $T$ be an operator on a Banach space $E$, and suppose that $y \otimes \lambda \in \overline{\mathscr{L}_{\{T\}}}$ for some $y \in E \backslash\{0\}$ and $\lambda \in E^{*}$. Then $\lambda \in \overline{T^{*}\left(E^{*}\right)}$.

Proof. Let $\left(S_{j}\right)_{j \in \mathbb{N}}$ be a sequence in $\mathscr{B}(E)$ such that $S_{j} T \rightarrow y \otimes \lambda$ as $j \rightarrow \infty$, and choose $\mu \in E^{*}$ such that $\langle y, \mu\rangle=1$. Then we have

$$
T^{*}\left(S_{j}^{*} \mu\right)=\left(S_{j} T\right)^{*} \mu \rightarrow(y \otimes \lambda)^{*} \mu=\langle y, \mu\rangle \lambda=\lambda \quad \text { as } \quad j \rightarrow \infty,
$$


from which the conclusion follows.

Example 4.10. Let $T$ be the operator on $\ell_{\infty}$ given by

$$
T\left(\alpha_{j}\right)_{j \in \mathbb{N}}=\left(-\frac{\alpha_{n}}{2^{n}}+\sum_{j=n+1}^{\infty} \frac{\alpha_{j}}{2^{j}}\right)_{n \in \mathbb{N}} \quad\left(\left(\alpha_{j}\right)_{j \in \mathbb{N}} \in \ell_{\infty}\right) .
$$

Then $T$ is compact and leaves the subspace $c_{0}$ invariant. Define $T_{0}: x \mapsto T x, c_{0} \rightarrow c_{0}$, and consider the closed left ideal $\mathscr{L}=\overline{\mathscr{L}_{\left\{T_{0}\right\}}}$ of $\mathscr{B}\left(c_{0}\right)$. We have $\mathscr{L} \subseteq \mathscr{K}\left(c_{0}\right)$ because $T_{0}$ is compact. Our aim is to show that $\mathscr{L}$ satisfies condition (a), but not condition (e), of Theorem 4.7.

We begin by verifying that $\operatorname{ker} T=\mathbb{C}(1,1, \ldots)$. It is clear that $T(1,1, \ldots)=(0,0, \ldots)$. Conversely, suppose that $\left(\alpha_{j}\right)_{j \in \mathbb{N}} \in \operatorname{ker} T$. Then, for each $n \in \mathbb{N}$, we have

$$
\frac{\alpha_{n}}{2^{n}}=\sum_{j=n+1}^{\infty} \frac{\alpha_{j}}{2^{j}}
$$

so that

$$
\frac{\alpha_{n}}{2^{n}}=\frac{\alpha_{n+1}}{2^{n+1}}+\sum_{j=n+2}^{\infty} \frac{\alpha_{j}}{2^{j}}=\frac{\alpha_{n+1}}{2^{n+1}}+\frac{\alpha_{n+1}}{2^{n+1}}=\frac{\alpha_{n+1}}{2^{n}} .
$$

Hence $\alpha_{n}=\alpha_{n+1}$ for each $n \in \mathbb{N}$, and the conclusion follows.

This shows in particular that $T_{0}$ is injective because $c_{0} \cap \operatorname{ker} T=\{0\}$. Consequently $T_{0} \notin \mathscr{M} \mathscr{L}_{x}$ for each $x \in c_{0} \backslash\{0\}$, and so $\mathscr{L}$ satisfies condition (a) of Theorem 4.7.

On the other hand, identifying $c_{0}^{* *}$ with $\ell_{\infty}$ in the usual way, we find that $T_{0}^{* *}=T$, which is not injective, so that $T_{0}^{*}$ does not have norm-dense range by [34, Theorem 3.1.17(b)]. Take $\lambda \in c_{0}^{*} \backslash \overline{T_{0}^{*}\left(c_{0}^{*}\right)}$ and $y \in E \backslash\{0\}$. Then, by Lemma 4.9, $y \otimes \lambda \notin \mathscr{L}$, so that $\mathscr{L}$ does not satisfy condition (e) of Theorem 4.7.

\section{5. 'Classical' Banach SPACES FOR WHich EACH Finitely-GENERATED, MAXimaL LEFT IDEAL IS FIXED}

The purpose of this section is to show that Question (II) has a positive answer for many standard examples of Banach spaces $E$.

We begin by showing that a much stronger conclusion is true in certain cases, namely that no finitely-generated, proper left ideal of $\mathscr{B}(E)$ contains $\mathscr{F}(E)$. This result relies on two lemmas. The first states that conditions (c)-(e) of Theorem 4.7 are equivalent for each finitely-generated left ideal $\mathscr{L}$ of $\mathscr{B}(E)$, whether or not $\mathscr{L}$ is closed.

Lemma 5.1. Let $E$ be a Banach space. Then the following three conditions are equivalent for each non-empty, finite subset $\Gamma$ of $\mathscr{B}(E)$ :

(a) the operator $\Psi_{\Gamma}$ is bounded below;

(b) the operator $\Omega_{\Gamma}$ is surjective;

(c) $\mathscr{L}_{\Gamma}$ contains $\mathscr{F}(E)$. 
Proof. The equivalence of (a) and (b) follows as in the final paragraph of the proof of Theorem 4.7.

To see that (b) and (c) are also equivalent, we note that Lemma 4.6(i) is true without the assumption that $\mathscr{L}_{\Gamma}$ is closed, provided that the set $\Gamma$ is finite, because in this case the series considered in the proof of Lemma 4.6(i), (c) $\Rightarrow(\mathrm{a})$, is finite. Hence Lemma 4.6(iii) also carries over to the present setting, and the conclusion follows.

Our second lemma characterizes the finite subsets $\Gamma$ of $\mathscr{B}(E)$ that do not generate a proper left ideal in terms of standard operator-theoretic properties of $\Psi_{\Gamma}$.

Lemma 5.2. Let $E$ be a non-zero Banach space. Then the following three conditions are equivalent for each non-empty, finite subset $\Gamma$ of $\mathscr{B}(E)$ :

(a) the operator $\Psi_{\Gamma}$ is bounded below and its range is complemented in $E^{|\Gamma|}$;

(b) the operator $\Psi_{\Gamma}$ is left invertible;

(c) $\mathscr{L}_{\Gamma}=\mathscr{B}(E)$.

Proof. The equivalence of (a) and (b) is an easy standard result, true for any operator between Banach spaces, while the equivalence of (b) and (c) follows immediately from (2.8).

Proposition 5.3. Let $E$ be a Banach space, and let $n \in \mathbb{N}$. Then $\mathscr{F}(E)$ is contained in a proper left ideal of $\mathscr{B}(E)$ generated by $n$ operators if and only if $E^{n}$ contains a closed subspace $F$ such that $F$ is isomorphic to $E$ and $F$ is not complemented in $E^{n}$.

Proof. We may suppose that $E$ is non-zero, and prove both implications by contraposition.

$\Rightarrow$. Suppose that each subspace $F$ of $E^{n}$ such that $F \cong E$ is automatically complemented, and let $\Gamma$ be a subset of $\mathscr{B}(E)$ of cardinality $n$ such that $\mathscr{F}(E) \subseteq \mathscr{L}_{\Gamma}$. We must prove that $\mathscr{L}_{\Gamma}=\mathscr{B}(E)$; that is, by Lemma 5.2, we must show that the operator $\Psi_{\Gamma}$ is bounded below and has complemented range. Lemma 5.1 implies that $\Psi_{\Gamma}$ is indeed bounded below, and its range is therefore a closed subspace of $E^{n}$ isomorphic to $E$, so that it is complemented by the assumption.

$\Leftarrow$. Suppose that $\mathscr{B}(E)$ is the only left ideal with (at most) $n$ generators that contains $\mathscr{F}(E)$, and let $F$ be a closed subspace of $E^{n}$ such that $F$ is isomorphic to $E$. We must prove that $F$ is complemented in $E^{n}$. Take an operator $T \in \mathscr{B}\left(E, E^{n}\right)$ which is bounded below and has range $F$. Letting $\Gamma=\left\{\rho_{1} T, \ldots, \rho_{n} T\right\} \subseteq \mathscr{B}(E)$, where

$$
\rho_{k}:\left(x_{j}\right)_{j=1}^{n} \mapsto x_{k}, \quad E^{n} \rightarrow E \quad(k \in\{1, \ldots, n\}),
$$

we have $\Psi_{\Gamma}=T$ by the definition (2.9) of $\Psi_{\Gamma}$. This operator is bounded below, and therefore Lemma 5.1 implies that $\mathscr{L}_{\Gamma}$ contains $\mathscr{F}(E)$. Hence, by the assumption, $\mathscr{L}_{\Gamma}=\mathscr{B}(E)$, so that the range of $\Psi_{\Gamma}$, which is equal to $F$, is complemented in $E^{n}$ by Lemma 5.2.

Combining this result with Theorem 1.1, we reach the following conclusion.

Corollary 5.4. Let $E$ be a Banach space such that, for each $n \in \mathbb{N}$, each closed subspace of $E^{n}$ isomorphic to $E$ is automatically complemented in $E^{n}$. Then $\mathscr{B}(E)$ is the only finitely-generated left ideal of $\mathscr{B}(E)$ which contains $\mathscr{F}(E)$, and hence each finitelygenerated, maximal left ideal of $\mathscr{B}(E)$ is fixed. 
Example 5.5. The condition of Corollary 5.4 on the Banach space $E$ is satisfied in each of the following three cases:

(i) $E$ is a Hilbert space.

(ii) $E$ is an injective Banach space; that is, whenever a Banach space $F$ contains a closed subspace $G$ which is isomorphic to $E$, then $G$ is automatically complemented in $F$. For instance, the Banach space $E=\ell_{\infty}(\Gamma)$ is injective for each non-empty set $\Gamma$. More generally, $E=C(K)$ is injective whenever the Hausdorff space $K$ is Stonean (that is, compact and extremely disconnected).

(iii) $E=c_{0}(\Gamma)$ for a non-empty set $\Gamma$ (this follows from Sobczyk's theorem [43] for countable $\Gamma$ and from [18] (or [3, Proposition 2.8]) in the general case); here $c_{0}(\Gamma)$ denotes the closed subspace of $\ell_{\infty}(\Gamma)$ consisting of those functions $f: \Gamma \rightarrow \mathbb{C}$ for which the set $\{\gamma \in \Gamma:|f(\gamma)| \geqslant \varepsilon\}$ is finite for each $\varepsilon>0$.

Thus, in each of these three cases, $\mathscr{B}(E)$ is the only finitely-generated left ideal of $\mathscr{B}(E)$ which contains $\mathscr{F}(E)$, and each finitely-generated, maximal left ideal of $\mathscr{B}(E)$ is fixed.

Our next goal is to prove a result (Theorem 5.9) which, under much less restrictive conditions on the Banach space $E$ than Corollary 5.4, gives the slightly weaker conclusion that $\mathscr{B}(E)$ is the only finitely-generated left ideal of $\mathscr{B}(E)$ which contains $\mathscr{K}(E)$. We note in particular that Corollary 4.2 ensures that this conclusion is still strong enough to ensure that each finitely-generated, maximal left ideal of $\mathscr{B}(E)$ is fixed, thus answering Question (II) positively for a large number of Banach spaces.

Let $E$ be a Banach space with a Schauder basis $\mathbf{e}=\left(e_{j}\right)_{j \in \mathbb{N}}$. For each $k \in \mathbb{N}$, we denote by $P_{k}$ the $k^{\text {th }}$ basis projection associated with $\mathbf{e}$. The basis constant of $\mathbf{e}$ is

$$
K_{\mathbf{e}}=\sup \left\{\left\|P_{k}\right\|: k \in \mathbb{N}\right\} \in[1, \infty) .
$$

The basis e is monotone if $K_{\mathbf{e}}=1$.

Lemma 5.6. Let $E$ be a Banach space with a Schauder basis $\mathbf{e}=\left(e_{j}\right)_{j \in \mathbb{N}}$, and let $\gamma=$ $\left(\gamma_{j}\right)_{j \in \mathbb{N}}$ be a decreasing sequence of non-negative real numbers. Then

$$
\Delta_{\gamma}: \sum_{j=1}^{\infty} \alpha_{j} e_{j} \mapsto \sum_{j=1}^{\infty} \gamma_{j} \alpha_{j} e_{j}
$$

defines an operator $\Delta_{\gamma} \in \mathscr{B}(E)$ of norm at most $K_{\mathbf{e}} \gamma_{1}$. This operator is compact if and only if $\gamma_{j} \rightarrow 0$ as $j \rightarrow \infty$.

Proof. Equation (5.1) clearly defines a linear mapping $\Delta_{\gamma}$ from $\operatorname{span}\left\{e_{j}: j \in \mathbb{N}\right\}$ into $E$. Since $\operatorname{span}\left\{e_{j}: j \in \mathbb{N}\right\}$ is dense in $E$, it suffices to show that this mapping is bounded with norm at most $K_{\mathbf{e}} \gamma_{1}$. Now, for each $x=\sum_{j=1}^{k} \alpha_{j} e_{j} \in \operatorname{span}\left\{e_{j}: j \in \mathbb{N}\right\}$, where $k \in \mathbb{N}$ and $\alpha_{1}, \ldots, \alpha_{k} \in \mathbb{C}$, we have

$$
\Delta_{\gamma} x=\gamma_{1} P_{1} x+\sum_{j=2}^{k} \gamma_{j}\left(P_{j} x-P_{j-1} x\right)=\sum_{j=1}^{k-1}\left(\gamma_{j}-\gamma_{j+1}\right) P_{j} x+\gamma_{k} x
$$


so that

$$
\left\|\Delta_{\gamma} x\right\| \leqslant \sum_{j=1}^{k-1}\left(\gamma_{j}-\gamma_{j+1}\right) K_{\mathbf{e}}\|x\|+\gamma_{k}\|x\| \leqslant K_{\mathbf{e}} \gamma_{1}\|x\|,
$$

as required.

To prove the final clause, we note that, by a standard result, $\left(P_{j}\right)_{j \in \mathbb{N}}$ is a bounded left approximate identity for $\mathscr{K}(E)$ (e.g., see [9, p. 318]), so that $\Delta_{\gamma}$ is compact if and only if $P_{j} \Delta_{\gamma} \rightarrow \Delta_{\gamma}$ as $j \rightarrow \infty$. Hence the estimates

$$
\gamma_{j+1} \leqslant\left\|\left(I_{E}-P_{j}\right) \Delta_{\gamma}\right\| \leqslant K_{\mathbf{e}}\left(K_{\mathbf{e}}+1\right) \gamma_{j+1} \quad(j \in \mathbb{N})
$$

which are easy to verify, give the result.

Corollary 5.7. Let $E$ be a Banach space with a Schauder basis $\mathbf{e}=\left(e_{j}\right)_{j \in \mathbb{N}}$, let $k \in \mathbb{N}$, and let $\eta=\left(\eta_{j}\right)_{j=1}^{k}$ be an increasing $k$-tuple of non-negative real numbers. Then

$$
\Theta_{\eta}: \sum_{j=1}^{\infty} \alpha_{j} e_{j} \mapsto \sum_{j=1}^{k} \eta_{j} \alpha_{j} e_{j}, \quad E \rightarrow E,
$$

defines an operator of norm at most $2 K_{\mathbf{e}} \eta_{k}$

Proof. Define

$$
f_{j}= \begin{cases}e_{k-j+1} & \text { for } j \leqslant k \\ e_{j} & \text { for } j>k\end{cases}
$$

Then $\mathbf{f}=\left(f_{j}\right)_{j \in \mathbb{N}}$ is a Schauder basis for $E$ (because we have reordered only finitely many vectors of the original basis $\mathbf{e}$ ), and the $m^{\text {th }}$ basis projection associated with $\mathbf{f}$ is given by $P_{k}-P_{k-m}$ for $m<k$ and $P_{m}$ for $m \geqslant k$, so that $K_{\mathbf{f}} \leqslant 2 K_{\mathbf{e}}$. Now Lemma 5.6 gives the desired conclusion because

$$
\Theta_{\eta}=\Delta_{\gamma}: \quad \sum_{j=1}^{\infty} \alpha_{j} f_{j} \mapsto \sum_{j=1}^{\infty} \gamma_{j} \alpha_{j} f_{j}, \quad E \rightarrow E,
$$

where $\gamma=\left(\gamma_{j}\right)_{j \in \mathbb{N}}$ denotes the decreasing sequence $\left(\eta_{k}, \eta_{k-1}, \ldots, \eta_{1}, 0,0, \ldots\right)$.

We now come to our key lemma.

Lemma 5.8. Let $E$ be a Banach space with a monotone Schauder basis, and let $\Gamma$ be a non-empty, finite subset of $\mathscr{B}(E)$ for which $\mathscr{F}(E) \subseteq \mathscr{L}_{\Gamma}$. Then the sequence $\left(t_{j}\right)_{j \in \mathbb{N}}$ given by

$$
t_{j}=\inf \left\{\|T\|: T \in \mathscr{B}\left(E^{|\Gamma|}, E\right), P_{j}=T \Psi_{\Gamma}\right\} \in(0, \infty) \quad(j \in \mathbb{N})
$$

is increasing. Suppose that $\left(t_{j}\right)_{j \in \mathbb{N}}$ is unbounded, and let $\gamma=\left(t_{j}^{-1 / 2}\right)_{j \in \mathbb{N}}$. Then the operator $\Delta_{\gamma}$ given by (5.1) is compact and does not belong to $\mathscr{L}_{\Gamma}$.

Proof. Set $n=|\Gamma| \in \mathbb{N}$. For each $j \in \mathbb{N}$, we have $P_{j} \in \mathscr{F}(E) \subseteq \mathscr{L}_{\Gamma}$, so that (2.8) ensures that the set appearing in the definition (5.3) of $t_{j}$ is non-empty, and $t_{j} \geqslant\left\|\Psi_{\Gamma}\right\|^{-1}>0$. To see that $t_{j+1} \geqslant t_{j}$, suppose that $P_{j+1}=T \Psi_{\Gamma}$ for some $T \in \mathscr{B}\left(E^{n}, E\right)$. Then $P_{j}=\left(P_{j} T\right) \Psi_{\Gamma}$, so that $t_{j} \leqslant\left\|P_{j} T\right\| \leqslant\|T\|$ by the monotonicity of the Schauder basis for $E$. 
The first part of the final clause (that $\Delta_{\gamma}$ is compact if $\left(t_{j}\right)_{j \in \mathbb{N}}$ is unbounded) is immediate from Lemma 5.6. We shall prove the second part by contraposition. Suppose that $\Delta_{\gamma} \in \mathscr{L}_{\Gamma}$, so that $\Delta_{\gamma}=S \Psi_{\Gamma}$ for some $S \in \mathscr{B}\left(E^{n}, E\right)$. Then, for each $k \in \mathbb{N}$, we have a commutative diagram

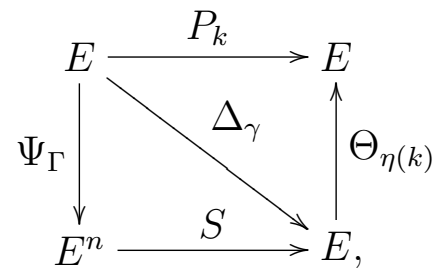

where $\eta(k)=\left(t_{j}^{1 / 2}\right)_{j=1}^{k}$ and the operator $\Theta_{\eta(k)}$ is given by (5.2). Hence, by the definition (5.3) of $t_{k}$ and Corollary 5.7, we obtain

$$
t_{k} \leqslant\left\|\Theta_{\eta(k)} S\right\| \leqslant 2 t_{k}^{1 / 2}\|S\|
$$

which implies that the sequence $\left(t_{j}\right)_{j \in \mathbb{N}}$ is bounded by $4\|S\|^{2}$.

Theorem 5.9. Let $E$ be a Banach space which is complemented in its bidual and has a Schauder basis. Then $\mathscr{B}(E)$ is the only finitely-generated left ideal of $\mathscr{B}(E)$ which contains $\mathscr{K}(E)$, and hence each finitely-generated, maximal left ideal of $\mathscr{B}(E)$ is fixed.

Proof. Let $\mathbf{e}=\left(e_{j}\right)_{j \in \mathbb{N}}$ be a Schauder basis for $E$. By passing to an equivalent norm on $E$, we may suppose that $\mathbf{e}$ is monotone. Suppose that $\Gamma$ is a non-empty, finite subset of $\mathscr{B}(E)$ such that $\mathscr{K}(E) \subseteq \mathscr{L}_{\Gamma}$, and set $n=|\Gamma| \in \mathbb{N}$. Lemma 5.8 implies that the sequence $\left(t_{j}\right)_{j \in \mathbb{N}}$ given by $(5.3)$ is bounded, so that we can find a bounded sequence $\left(T_{j}\right)_{j \in \mathbb{N}}$ in $\mathscr{B}\left(E^{n}, E\right)$ such that $P_{j}=T_{j} \Psi_{\Gamma}$ for each $j \in \mathbb{N}$.

We may identify $\mathscr{B}\left(E^{n}, E^{* *}\right)$ with the dual space of the projective tensor product $E^{n} \widehat{\otimes} E^{*}$; the duality bracket is given by

$$
\langle x \otimes \lambda, S\rangle=\langle\lambda, S x\rangle \quad\left(x \in E^{n}, \lambda \in E^{*}, S \in \mathscr{B}\left(E^{n}, E^{* *}\right)\right)
$$

(e.g., see [9, Proposition A.3.70]). Hence $\mathscr{B}\left(E^{n}, E^{* *}\right)$ carries a weak ${ }^{*}$-topology, with respect to which its unit ball is compact by the Banach-Alaoglu theorem. Consequently the bounded sequence $\left(\kappa_{E} T_{j}\right)_{j \in \mathbb{N}}$ has a weak*-accumulation point, say $T \in \mathscr{B}\left(E^{n}, E^{* *}\right)$. Then, for each $j \in \mathbb{N}, \lambda \in E^{*}$ and $\varepsilon>0$, we can find an integer $k \geqslant j$ such that

$$
\varepsilon \geqslant\left|\left\langle\Psi_{\Gamma} e_{j} \otimes \lambda, T-\kappa_{E} T_{k}\right\rangle\right|=\left|\left\langle\lambda, T \Psi_{\Gamma} e_{j}-\kappa_{E} T_{k} \Psi_{\Gamma} e_{j}\right\rangle\right|=\left|\left\langle\lambda, T \Psi_{\Gamma} e_{j}-\kappa_{E} e_{j}\right\rangle\right| .
$$

Since $\varepsilon>0$ and $\lambda \in E^{*}$ were arbitrary, we conclude that $T \Psi_{\Gamma} e_{j}=\kappa_{E} e_{j}$, and therefore $T \Psi_{\Gamma}=\kappa_{E}$. By the assumption, $\kappa_{E}$ has a left inverse, say $\Lambda \in \mathscr{B}\left(E^{* *}, E\right)$. Hence we have $I_{E}=(\Lambda T) \Psi_{\Gamma} \in \mathscr{L}_{\Gamma}$, so that $\mathscr{L}_{\Gamma}=\mathscr{B}(E)$.

Example 5.10. Theorem 5.9 implies that, for $E=\ell_{p}$ or $E=L_{p}[0,1]$, where $p \in(1, \infty)$, $\mathscr{B}(E)$ is the only finitely-generated left ideal of $\mathscr{B}(E)$ which contains $\mathscr{K}(E)$, and each finitely-generated, maximal left ideal of $\mathscr{B}(E)$ is fixed. This conclusion is also true for $p=1$; indeed, $\ell_{1}$ is a dual space and therefore complemented in its bidual, while $L_{1}[0,1]$ is complemented in its bidual by [1, Theorem 6.3.10]. 
Many other Banach spaces are known to be complemented in their biduals. The following list gives some examples.

(i) Let $E$ be a Banach space which is isomorphic to a complemented subspace of a dual Banach space; that is, for some Banach space $F$, there are operators $U \in \mathscr{B}\left(E, F^{*}\right)$ and $V \in \mathscr{B}\left(F^{*}, E\right)$ such that $I_{E}=V U$. Then the diagram

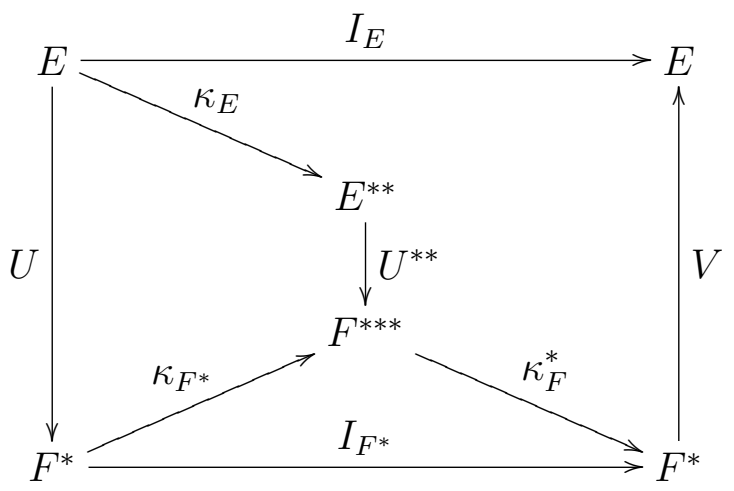

is commutative. This implies that the operator $\kappa_{E} V \kappa_{F}^{*} U^{* *}$ is a projection of $E^{* *}$ onto $\kappa_{E}(E)$, so that $E$ is complemented in its bidual.

(ii) Let $E$ be a Banach lattice which does not contain a subspace isomorphic to $c_{0}$. Then $E$ is complemented in its bidual by [32, Theorem 1.c.4].

(iii) Let $E$ be a non-zero Banach space for which $\mathscr{B}(E)$ is complemented in its bidual, so that $I_{\mathscr{B}(E)}=\Lambda \kappa_{\mathscr{B}(E)}$ for some operator $\Lambda \in \mathscr{B}\left(\mathscr{B}(E)^{* *}, \mathscr{B}(E)\right)$. Choosing $\lambda \in E^{*}$ and $y \in E$ with $\langle y, \lambda\rangle=1$, we can define operators by

$$
U_{\lambda}: x \mapsto x \otimes \lambda, \quad E \rightarrow \mathscr{B}(E), \quad \text { and } \quad V_{y}: T \mapsto T y, \quad \mathscr{B}(E) \rightarrow E,
$$

which induce a commutative diagram

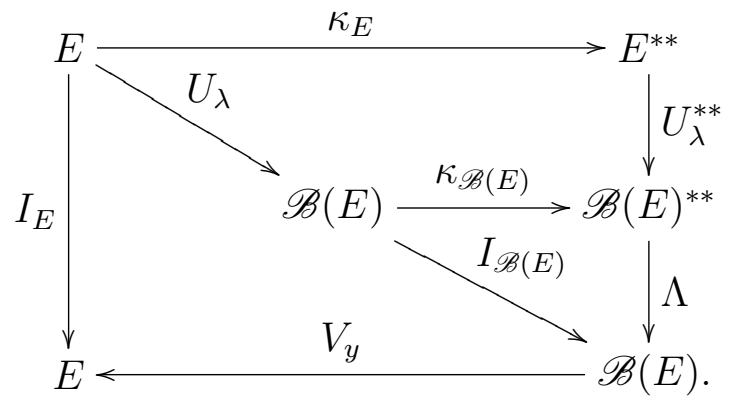

This shows that $V_{y} \Lambda U_{\lambda}^{* *}$ is a left inverse of $\kappa_{E}$, and so $E$ is complemented in its bidual.

Remark 5.11. Theorem 5.9 does not provide any new information for Banach spaces of the form $E=C(K)$, where $K$ is a compact Hausdorff space, because the assumption that $C(K)$ is complemented in its bidual implies that $C(K)$ is injective, so that Example 5.5(ii) already applies.

A slight variation of the proof of Theorem 5.9 gives the following conclusion. 
Theorem 5.12. Let $E$ be a Banach space with a Schauder basis. Then $\mathscr{B}\left(E^{*}\right)$ is the only finitely-generated left ideal of $\mathscr{B}\left(E^{*}\right)$ which contains $\overline{\mathscr{F}\left(E^{*}\right)}$, and hence each finitelygenerated, maximal left ideal of $\mathscr{B}\left(E^{*}\right)$ is fixed.

Proof. (Sketch.) Let $\Gamma$ be a non-empty, finite subset of $\mathscr{B}\left(E^{*}\right)$ such that $\overline{\mathscr{F}\left(E^{*}\right)} \subseteq \mathscr{L}_{\Gamma}$, and set $n=|\Gamma| \in \mathbb{N}$. As in the proof of Theorem 5.9, we may suppose that $E$ has a monotone Schauder basis e. Then, arguing as in the proof of Lemma 5.8, we see that the sequence

$$
t_{j}^{\prime}=\inf \left\{\|T\|: T \in \mathscr{B}\left(\left(E^{*}\right)^{n}, E^{*}\right), P_{j}^{*}=T \Psi_{\Gamma}\right\} \in(0, \infty) \quad(j \in \mathbb{N})
$$

is increasing and bounded. (Indeed, if $\left(t_{j}^{\prime}\right)_{j \in \mathbb{N}}$ were unbounded, then for $\gamma=\left(\left(t_{j}^{\prime}\right)^{-1 / 2}\right)_{j \in \mathbb{N}}$, we would have $\Delta_{\gamma}^{*} \in \overline{\mathscr{F}\left(E^{*}\right)} \backslash \mathscr{L}_{\Gamma}$, contrary to our assumption.) Consequently there exists a bounded sequence $\left(T_{j}\right)_{j \in \mathbb{N}}$ in $\mathscr{B}\left(\left(E^{*}\right)^{n}, E^{*}\right)$ such that $P_{j}^{*}=T_{j} \Psi_{\Gamma}$ for each $j \in \mathbb{N}$. Let $T$ be a weak*-accumulation point of $\left(T_{j}\right)_{j \in \mathbb{N}}$, where we have identified $\mathscr{B}\left(\left(E^{*}\right)^{n}, E^{*}\right)$ with the dual space of the projective tensor product $E \widehat{\otimes}\left(E^{*}\right)^{n}$ via the duality bracket given by

$$
\langle x \otimes \mu, S\rangle=\langle x, S \mu\rangle \quad\left(x \in E, \mu \in\left(E^{*}\right)^{n}, S \in \mathscr{B}\left(\left(E^{*}\right)^{n}, E^{*}\right)\right) .
$$

For each $x \in E, \lambda \in E^{*}$, and $\varepsilon>0$, we can find $j_{0} \in \mathbb{N}$ such that $\left\|x-P_{j} x\right\| \leqslant \varepsilon(2\|\lambda\|+1)^{-1}$ whenever $j \geqslant j_{0}$. Choosing $j \geqslant j_{0}$ such that $\left|\left\langle x \otimes \Psi_{\Gamma} \lambda, T-T_{j}\right\rangle\right| \leqslant \varepsilon / 2$ and applying (5.4), we then obtain

$$
\begin{aligned}
\left|\left\langle x,\left(T \Psi_{\Gamma}-I_{E^{*}}\right) \lambda\right\rangle\right| & \leqslant\left|\left\langle x,\left(T \Psi_{\Gamma}-P_{j}^{*}\right) \lambda\right\rangle\right|+\left|\left\langle x,\left(I_{E}-P_{j}\right)^{*} \lambda\right\rangle\right| \\
& \leqslant\left|\left\langle x,\left(T-T_{j}\right) \Psi_{\Gamma} \lambda\right\rangle\right|+\left|\left\langle x-P_{j} x, \lambda\right\rangle\right| \leqslant \varepsilon .
\end{aligned}
$$

This implies that $T \Psi_{\Gamma}=I_{E^{*}}$, and therefore $\mathscr{L}_{\Gamma}=\mathscr{B}\left(E^{*}\right)$.

Example 5.13. Theorem 5.12 applies in the following two cases which have not already been resolved:

(i) $E=X \widehat{\otimes} X^{*}$, where $X$ is a Banach space with a shrinking Schauder basis (this ensures that $E$ has a Schauder basis); then $E^{*} \cong \mathscr{B}\left(X^{*}\right)$, so that the conclusion is that each finitely-generated, maximal left ideal of $\mathscr{B}\left(\mathscr{B}\left(X^{*}\right)\right)$ is fixed. The most important case is where $X$, and hence $X^{*}$, is a separable, infinite-dimensional Hilbert space; in this case $\mathscr{B}\left(X^{*}\right)$ does not have the approximation property [44].

(ii) $E=\left(\bigoplus_{n \in \mathbb{N}} E_{n}\right)_{\ell_{1}}$, where $\left(E_{n}\right)_{n \in \mathbb{N}}$ is a sequence of Banach spaces with Schauder bases whose basis constants are uniformly bounded; then $E^{*} \cong\left(\bigoplus_{n \in \mathbb{N}} E_{n}^{*}\right)_{\ell_{\infty}}$, and so the conclusion is that each finitely-generated, maximal left ideal of $\mathscr{B}\left(\left(\bigoplus E_{n}^{*}\right)_{\ell_{\infty}}\right)$ is fixed.

The conditions imposed on the Banach space $E$ in Theorems 5.9 and 5.12 are clearly preserved under the formation of finite direct sums. By contrast, this need not be the case for the condition of Corollary 5.4. For instance, $c_{0}$ and $\ell_{\infty}$ both satisfy this condition by Example 5.5(ii)-(iii), whereas their direct sum $c_{0} \oplus \ell_{\infty}$ does not. We shall explore this situation in greater depth in Section 7. Notably, as a particular instance of Theorem 7.3, we shall see that the main conclusion of Corollary 5.4 fails for $E=c_{0} \oplus \ell_{\infty}$ because $\mathscr{F}\left(c_{0} \oplus \ell_{\infty}\right)$ is contained in a proper, closed, singly-generated left ideal of $\mathscr{B}\left(c_{0} \oplus \ell_{\infty}\right)$. 
We do not know the answer to Question (II) for $E=c_{0} \oplus \ell_{\infty}$, but the following result answers this question positively for another direct sum arising naturally from Example 5.5, with the ideal $\mathscr{S}(E)$ of strictly singular operators taking the role that was played by $\mathscr{F}(E)$ in Corollary 5.4 and $\mathscr{K}(E)$ in Theorem 5.9.

Proposition 5.14. Let $E=c_{0}(\Gamma) \oplus H$, where $\Gamma$ is a non-empty set and $H$ is a Hilbert space. Then $\mathscr{B}(E)$ is the only finitely-generated left ideal of $\mathscr{B}(E)$ which contains $\mathscr{S}(E)$, and hence each finitely-generated, maximal left ideal of $\mathscr{B}(E)$ is fixed.

Proof. Let $\mathscr{L}$ be a finitely-generated left ideal of $\mathscr{B}(E)$ such that $\mathscr{S}(E) \subseteq \mathscr{L}$. We may suppose that $\Gamma$ is infinite and $H$ is infinite-dimensional. Proposition 2.1 implies that $\mathscr{L}$ is generated by a single operator $T \in \mathscr{B}(E)$, say, while Lemma 5.1 shows that $T$ is bounded below and thus is an upper semi-Fredholm operator.

We can represent $T$ as a matrix of operators:

$$
T=\left(\begin{array}{cc}
T_{1,1}: c_{0}(\Gamma) \rightarrow c_{0}(\Gamma) & T_{1,2}: H \rightarrow c_{0}(\Gamma) \\
T_{2,1}: c_{0}(\Gamma) \rightarrow H & T_{2,2}: H \rightarrow H
\end{array}\right)
$$

Each operator from $H$ to $c_{0}(\Gamma)$ is strictly singular because no infinite-dimensional subspace of $c_{0}(\Gamma)$ is isomorphic to a Hilbert space. Similarly, each operator from $c_{0}(\Gamma)$ to $H$ is strictly singular. Hence, by (2.6), we obtain

$$
\left(\begin{array}{cc}
T_{1,1} & 0 \\
0 & T_{2,2}
\end{array}\right)=T-\left(\begin{array}{cc}
0 & T_{1,2} \\
T_{2,1} & 0
\end{array}\right) \in \Phi_{+}(E)
$$

which clearly implies that $T_{1,1} \in \Phi_{+}\left(c_{0}(\Gamma)\right)$ and $T_{2,2} \in \Phi_{+}(H)$. Let $P_{1} \in \mathscr{F}\left(c_{0}(\Gamma)\right)$ and $P_{2} \in \mathscr{F}(H)$ be projections onto the kernels of $T_{1,1}$ and $T_{2,2}$, respectively. Then

$$
\widetilde{T}_{1,1}: x \mapsto T_{1,1} x, \quad \text { ker } P_{1} \rightarrow T_{1,1}\left(c_{0}(\Gamma)\right),
$$

is an isomorphism, and we have $T_{1,1}\left(c_{0}(\Gamma)\right) \cong \operatorname{ker} P_{1} \cong c_{0}(\Gamma)$ because $c_{0}(\Gamma)$ is isomorphic to its hyperplanes. Consequently, as in Example 5.5(iii), the range of $T_{1,1}$ is complemented in $c_{0}(\Gamma)$, so that we can extend the inverse of $\widetilde{T}_{1,1}$ to obtain an operator $S_{1} \in \mathscr{B}\left(c_{0}(\Gamma)\right)$ which satisfies $S_{1} T_{1,1}=I_{c_{0}(\Gamma)}-P_{1}$. Similarly, we can find an operator $S_{2} \in \mathscr{B}(H)$ such that $S_{2} T_{2,2}=I_{H}-P_{2}$. In conclusion, we have

$$
I_{E}=\left(\begin{array}{cc}
S_{1} & 0 \\
0 & S_{2}
\end{array}\right) T+\left(\begin{array}{cc}
P_{1} & -S_{1} T_{1,2} \\
-S_{2} T_{2,1} & P_{2}
\end{array}\right) \in \mathscr{L}+\mathscr{S}(E)=\mathscr{L},
$$

and thus $\mathscr{L}=\mathscr{B}(E)$.

The final clause follows immediately from Corollary 4.2 because $\mathscr{S}(E) \subseteq \mathscr{E}(E)$.

\section{6. 'EXOTIC' BANACH SPACES FOR WHICH EACH FINITELY-GENERATED, MAXIMAL LEFT IDEAL IS FIXED}

In this section, we shall answer Question (II) positively for two classes of custom-made Banach spaces of a distinctly non-classical nature, using an approach which is completely different from the one taken in Section 5. More precisely, for each Banach space $E$ in either of these two classes, we are able to describe all the maximal left ideals of $\mathscr{B}(E)$ explicitly, 
and it will then follow easily that only the fixed maximal left ideals are finitely generated. The reason that we can describe all the maximal left ideals of $\mathscr{B}(E)$ is, roughly speaking, that $\mathscr{B}(E)$ is 'small'. As we shall see, in both cases each non-fixed, maximal left ideal of $\mathscr{B}(E)$ is a two-sided ideal of codimension one.

We begin with a lemma which can be viewed as a counterpart of Corollary 4.8 for left ideals of strictly singular operators.

Lemma 6.1. Let $E$ be a Banach space, and let $\mathscr{L}$ be a left ideal of $\mathscr{B}(E)$ such that $\mathscr{F}(E) \subseteq \mathscr{L} \subseteq \mathscr{S}(E)$. Then the following three conditions are equivalent:

(a) $\mathscr{L}$ is finitely generated;

(b) $\mathscr{L}=\mathscr{B}(E)$;

(c) E is finite-dimensional.

Proof. The implications $(\mathrm{c}) \Rightarrow(\mathrm{b}) \Rightarrow(\mathrm{a})$ are clear.

(a) $\Rightarrow$ (c). Suppose that $\mathscr{L}=\mathscr{L}_{\Gamma}$ for some non-empty, finite subset $\Gamma$ of $\mathscr{B}(E)$. Lemma 5.1 implies that the operator $\Psi_{\Gamma}$ is bounded below, while (2.9) and the fact that $\Gamma \subseteq \mathscr{S}(E)$ show that $\Psi_{\Gamma}$ is strictly singular. Hence the domain $E$ of $\Psi_{\Gamma}$ is finite-dimensional.

A Banach space $E$ has few operators if $E$ is infinite-dimensional and each operator on $E$ is the sum of a scalar multiple of the identity operator and a strictly singular operator; that is, $\mathscr{B}(E)=\mathbb{C} I_{E}+\mathscr{S}(E)$. Gowers and Maurey [16] showed that each hereditarily indecomposable Banach space has few operators, and constructed the first example of such a space.

Theorem 6.2. Let $E$ be a Banach space which has few operators. Then $\mathscr{S}(E)$ is the unique non-fixed, maximal left ideal of $\mathscr{B}(E)$, and $\mathscr{S}(E)$ is not finitely generated as a left ideal.

Proof. Let $\mathscr{L}$ be a maximal left ideal of $\mathscr{B}(E)$, and suppose that $\mathscr{L}$ is not fixed. Then, by Corollary $4.2, \mathscr{L}$ contains $\mathscr{E}(E)$ and hence $\mathscr{S}(E)$, which has codimension one in $\mathscr{B}(E)$, so that $\mathscr{L}=\mathscr{E}(E)=\mathscr{S}(E)$. This proves the first clause. The second clause follows from Lemma 6.1.

To set the scene for our second result, we begin with a short excursion into the theory of semidirect products of Banach algebras. Let $\mathscr{B}$ be a Banach algebra, and let $\mathscr{C}$ and $\mathscr{I}$ be a closed subalgebra and a closed, two-sided ideal of $\mathscr{B}$, respectively. Then $\mathscr{B}$ is the semidirect product of $\mathscr{C}$ and $\mathscr{I}$ if $\mathscr{C}$ and $\mathscr{I}$ are complementary subspaces of $\mathscr{B}$; that is, $\mathscr{C}+\mathscr{I}=\mathscr{B}$ and $\mathscr{C} \cap \mathscr{I}=\{0\}$. In this case, we denote by $\rho: \mathscr{B} \rightarrow \mathscr{C}$ the projection of $\mathscr{B}$ onto $\mathscr{C}$ along $\mathscr{I}$. This is an algebra homomorphism, as is easy to check. It is relevant for our purposes because it induces an isomorphism between the lattices of closed left ideals

$$
\text { Lat }_{\mathscr{I}}(\mathscr{B})=\{\mathscr{L}: \mathscr{L} \text { is a closed left ideal of } \mathscr{B} \text { such that } \mathscr{I} \subseteq \mathscr{L}\}
$$

and

$$
\operatorname{Lat}(\mathscr{C})=\{\mathscr{N}: \mathscr{N} \text { is a closed left ideal of } \mathscr{C}\} .
$$

More precisely, for each $\mathscr{L} \in \operatorname{Lat}_{\mathscr{I}}(\mathscr{B})$, we have $\rho(\mathscr{L})=\mathscr{L} \cap \mathscr{C} \in \operatorname{Lat}(\mathscr{C})$, and the mapping $\mathscr{L} \mapsto \rho(\mathscr{L})$ is a lattice isomorphism of $\operatorname{Lat} \mathscr{I}(\mathscr{B})$ onto $\operatorname{Lat}(\mathscr{C})$; its inverse is given 
by $\mathscr{N} \mapsto \mathscr{N}+\mathscr{I}$. Suppose that the left ideal $\mathscr{L} \in \operatorname{Lat}_{\mathscr{I}}(\mathscr{B})$ is generated by a subset $\Gamma$ of $\mathscr{B}$. Then evidently $\rho(\mathscr{L})$ is generated by the subset $\rho(\Gamma)$ of $\mathscr{C}$, so that $\rho$ maps each closed, finitely-generated left ideal of $\mathscr{B}$ containing $\mathscr{I}$ to a closed, finitely-generated left ideal of $\mathscr{C}$.

We shall next state two classical results about $C(K)$-spaces. The first is due to Pełczyński [36, Theorem 1], and characterizes the weakly compact operators from a $C(K)$-space into an arbitrary Banach space.

Theorem 6.3. Let $K$ be a compact Hausdorff space, and let $E$ be a Banach space. Then the following three conditions are equivalent for each operator $T \in \mathscr{B}(C(K), E)$ :

(a) $T$ is weakly compact;

(b) $T$ is strictly singular;

(c) $T$ does not fix a copy of $c_{0}$.

The second result describes the maximal ideals of the Banach algebra $C(K)$, as well as the finitely-generated ones. (Note that the notions of a left, right, and two-sided ideal coincide in $C(K)$ because $C(K)$ is commutative.) Given a point $k \in K$, we write $\varepsilon_{k}: C(K) \rightarrow \mathbb{C}$ for the evaluation map at $k$; that is, $\varepsilon_{k}(f)=f(k)$ for each $f \in C(K)$. This is a surjective algebra homomorphism of norm one.

Theorem 6.4. Let $K$ be a compact Hausdorff space. Then:

(i) each maximal ideal of $C(K)$ has the form $\operatorname{ker} \varepsilon_{k}$ for a unique point $k \in K$;

(ii) the maximal ideal ker $\varepsilon_{k}$ is finitely generated if and only if $k$ is isolated in $K$.

Proof. The first clause is folklore (e.g., see [9, Theorem 4.2.1(i)]), while the second is the complex-valued counterpart of a classical theorem of Gillman [14, Corollary 5.4]. Both clauses are also easy to verify directly.

We require one further notion before we can present our result. For a compact Hausdorff space $K$ and a function $g \in C(K)$, we denote by $M_{g} \in \mathscr{B}(C(K))$ the multiplication operator given by $g$; that is, $M_{g} f=g f$ for each $f \in C(K)$. The mapping

$$
\mu: g \mapsto M_{g}, \quad C(K) \rightarrow \mathscr{B}(C(K)),
$$

is an isometric, unital algebra homomorphism. An operator $T \in \mathscr{B}(C(K))$ is a weak multiplication if it has the form $T=M_{g}+S$ for some $g \in C(K)$ and $S \in \mathscr{W}(C(K))$. The fourth-named author [27, Theorem 6.1] (assuming the continuum hypothesis) and Plebanek [37, Theorem 1.3] (without any assumptions beyond ZFC) have constructed an example of a connected, compact Hausdorff space $K$ for which each operator on $C(K)$ is a weak multiplication. This ensures that the following theorem is not vacuous.

Theorem 6.5. Let $K$ be a compact Hausdorff space without isolated points and such that each operator on $C(K)$ is a weak multiplication.

(i) The Banach algebra $\mathscr{B}(C(K))$ is the semidirect product of the subalgebra $\mu(C(K))$ and the ideal $\mathscr{W}(C(K))$, where $\mu$ is the homomorphism given by (6.3).

(ii) The following four conditions are equivalent for each subset $\mathscr{L}$ of $\mathscr{B}(C(K))$ : 
(a) $\mathscr{L}$ is a non-fixed, maximal left ideal of $\mathscr{B}(C(K))$;

(b) $\mathscr{L}$ is a maximal left ideal of $\mathscr{B}(C(K))$, and $\mathscr{L}$ is not finitely generated;

(c) $\mathscr{L}$ is a maximal two-sided ideal of $\mathscr{B}(C(K))$;

(d) $\mathscr{L}=\left\{M_{g}+S: S \in \mathscr{W}(C(K))\right.$ and $g \in C(K)$ with $\left.g(k)=0\right\}$ for some $k \in K$.

In the positive case, the point $k \in K$ such that (d) holds is uniquely determined by $\mathscr{L}$.

Proof. (i). We have $\mathscr{B}(C(K))=\mu(C(K))+\mathscr{W}(C(K))$ because each operator on $C(K)$ is a weak multiplication. Theorem 6.3 allows us to replace $\mathscr{W}(C(K))$ with $\mathscr{S}(C(K))$, which we shall do in the remainder of this proof because the latter ideal suits our approach better.

To see that $\mu(C(K)) \cap \mathscr{S}(C(K))=\{0\}$, suppose that $g \in C(K) \backslash\{0\}$. Take $k_{0} \in K$ such that $g\left(k_{0}\right) \neq 0$, set $\varepsilon=\left|g\left(k_{0}\right)\right| / 2>0$, and choose an open neighbourhood $N$ of $k_{0}$ such that $|g(k)| \geqslant \varepsilon$ for each $k \in N$. Using Urysohn's lemma and the fact that $k_{0}$ is not isolated in $K$, we deduce that the subspace

$$
F=\{f \in C(K): f(k)=0 \quad(k \in K \backslash N)\}
$$

of $C(K)$ is infinite dimensional. Since

$$
\left\|M_{g} f\right\|=\sup \{|g(k) f(k)|: k \in N\} \geqslant \varepsilon\|f\| \quad(f \in F),
$$

we conclude that $M_{g}$ is not strictly singular, as required.

(ii). For each $k \in K$, let

$$
\mathscr{Z}_{k}=\mu\left(\operatorname{ker} \varepsilon_{k}\right)+\mathscr{S}(C(K))=\left\{M_{g}+S: S \in \mathscr{S}(C(K)) \text { and } g \in C(K) \text { with } g(k)=0\right\} \text {. }
$$

By (i), $\mathscr{Z}_{k}$ is a two-sided ideal of codimension one in $\mathscr{B}(C(K))$, and thus maximal both as a left and a two-sided ideal. The implication $(\mathrm{d}) \Rightarrow(\mathrm{c})$ is now immediate, while $(\mathrm{d}) \Rightarrow(\mathrm{b})$ follows because $\rho\left(\mathscr{Z}_{k}\right)=\mu\left(\operatorname{ker} \varepsilon_{k}\right)$ is not finitely generated by Theorem $6.4(\mathrm{ii})$, so that $\mathscr{Z}_{k}$ is not finitely generated as a left ideal, as explained in the paragraph following (6.2).

The implication $(b) \Rightarrow(a)$ is clear because each fixed, maximal left ideal is finitely generated by Proposition 2.2(i).

(a) $\Rightarrow($ d). Suppose that $\mathscr{L}$ is a non-fixed, maximal left ideal of $\mathscr{B}(C(K))$. Then, by Corollary $4.2, \mathscr{L}$ contains $\mathscr{E}(C(K))$ and thus $\mathscr{S}(C(K))$, so that $\mathscr{L}$ is a maximal element of the lattice Lat $\operatorname{S}_{(C(K))}(\mathscr{B}(C(K)))$ given by (6.1). Hence, in the notation of (6.2), there is a maximal element $\mathscr{N}$ of the lattice $\operatorname{Lat}(\mu(C(K)))$ such that $\mathscr{L}=\mathscr{N}+\mathscr{S}(C(K))$. Theorem 6.4(i) implies that $\mathscr{N}=\mu\left(\operatorname{ker} \varepsilon_{k}\right)$ for some $k \in K$, and consequently $\mathscr{L}=\mathscr{Z}_{k}$.

$(\mathrm{c}) \Rightarrow(\mathrm{d})$. Suppose that $\mathscr{L}$ is a maximal two-sided ideal of $\mathscr{B}(C(K))$. Then, as mentioned in Remark 4.3, $\mathscr{L}$ contains $\mathscr{E}(C(K))$ and hence $\mathscr{S}(C(K))$, so that $\mathscr{L}=\mathscr{Z}_{k}$ for some $k \in K$ by (i) and Theorem 6.4(i).

The final clause follows because $\operatorname{ker} \varepsilon_{k_{1}} \neq \operatorname{ker} \varepsilon_{k_{2}}$ whenever $k_{1}, k_{2} \in K$ are distinct, and hence also $\mathscr{Z}_{k_{1}} \neq \mathscr{Z}_{k_{2}}$.

Remark 6.6. Example 5.5(ii)-(iii) and Theorem 6.5 show that, for certain compact Hausdorff spaces $K$, Question (II) has a positive answer for $E=C(K)$. However, this question remains open for some very important $C(K)$-spaces. Indeed, it is known that $C(K)$ contains a closed subspace which is isomorphic to $C(K)$ and which is not complemented in $C(K)$ for each of the following compact Hausdorff spaces $K$ : 
(i) $K=[0,1]$ (see $[2])$;

(ii) $K=[0, \alpha]$ for any ordinal $\alpha \geqslant \omega^{\omega}$, where $[0, \alpha]$ denotes the set of ordinals less than or equal to $\alpha$, equipped with the order topology. (Baker [5] showed this for $\alpha=\omega^{\omega}$; the conclusion for general $\alpha \geqslant \omega^{\omega}$ follows immediately from Baker's result because $\left.C[0, \alpha] \cong C[0, \alpha] \oplus C\left[0, \omega^{\omega}\right].\right)$

Hence, by Lemma 5.1, $\mathscr{F}(C(K))$ is contained in a singly-generated, proper left ideal of $\mathscr{B}(C(K))$ for each of these $K$, but we do not know whether such a left ideal can be chosen also to be maximal (or even closed).

This question cannot be answered by a variant of Theorem 5.9 because we can strengthen the above conclusion to obtain that $\mathscr{K}(C(K))$ is contained in a singly-generated, proper left ideal of $\mathscr{B}(C(K))$ for each of the above $K$. To see this, take an operator $U \in \mathscr{B}(C(K))$ which is bounded below and whose range $F=U(C(K))$ is not complemented in $C(K)$, and consider the isomorphism $\widetilde{U}: x \mapsto U x, C(K) \rightarrow F$. Then, for each $S \in \mathscr{K}(C(K))$, the operator $S \widetilde{U}^{-1}: F \rightarrow C(K)$ has an extension $T \in \mathscr{K}(C(K))$ by a theorem of Grothendieck (see [19, pp. 559-560], or [30, Theorem 1]). Hence we have $S=T U$, and consequently $\mathscr{K}(C(K)) \subseteq \mathscr{L}_{\{U\}}$.

\section{A NON-FIXED AND SINGLY-GENERATED, MAXIMAL LEFT IDEAL OF OPERATORS}

The main aim of this section is to prove Theorems 1.5 and 1.6. Several parts of those theorems are special cases of more general results, which may be of independent interest, and so we shall take a more general approach, specializing only when we need to.

Recall that, for a non-empty set $\Gamma$, we denote by $\ell_{\infty}(\Gamma)$ the Banach space of bounded, complex-valued functions defined on $\Gamma$, and $\ell_{\infty}=\ell_{\infty}(\mathbb{N})$. Our first result collects some known facts about operators from $\ell_{\infty}(\Gamma)$ that we shall use several times.

Lemma 7.1. Let $\Gamma$ be a non-empty set, and let $X$ be a Banach space.

(i) An operator from $\ell_{\infty}(\Gamma)$ to $X$ is weakly compact if and only if it is strictly singular.

(ii) Suppose that the set $\Gamma$ is infinite. Then each operator from $\ell_{\infty}(\Gamma)$ to $X$ is weakly compact if and only if $X$ does not contain a subspace isomorphic to $\ell_{\infty}$.

Proof. (i). This is a special case of Theorem 6.3.

(ii). The hard part is the implication $\Leftarrow$, which however follows immediately from [31, Proposition 2.f.4].

The forward implication is straightforward. Suppose contrapositively that $X$ contains a subspace isomorphic to $\ell_{\infty}$, and take an operator $U \in \mathscr{B}\left(\ell_{\infty}, X\right)$ which is bounded below. Choose an injective mapping $\theta: \mathbb{N} \rightarrow \Gamma$, and define an operator $C_{\theta} \in \mathscr{B}\left(\ell_{\infty}(\Gamma), \ell_{\infty}\right)$ by $C_{\theta} f=f \circ \theta$ for each $f \in \ell_{\infty}(\Gamma)$. Then $U C_{\theta}$ is not weakly compact, for instance because it fixes a copy of $\ell_{\infty}$.

In the remainder of this section we shall consider a Banach space $X$ such that

(I) the bidual of $X$ is isomorphic to $\ell_{\infty}\left(\Upsilon_{1}\right)$ for some infinite set $\Upsilon_{1}$ via a fixed isomorphism $V: X^{* *} \rightarrow \ell_{\infty}\left(\Upsilon_{1}\right)$; and

(II) no subspace of $X$ is isomorphic to $\ell_{\infty}$. 
For example, $X=c_{0}$ satisfies both of these conditions with $\Upsilon_{1}=\mathbb{N}$.

Let $\Upsilon_{2}$ be a disjoint copy of $\Upsilon_{1}$ (that is, $\Upsilon_{2}$ is a set of the same cardinality as $\Upsilon_{1}$ and satisfies $\left.\Upsilon_{1} \cap \Upsilon_{2}=\emptyset\right)$, and set $\Upsilon=\Upsilon_{1} \cup \Upsilon_{2}$. We consider $\ell_{\infty}\left(\Upsilon_{1}\right)$ and $\ell_{\infty}\left(\Upsilon_{2}\right)$ as complementary subspaces of $\ell_{\infty}(\Upsilon)$ in the natural way, and denote by $P_{1}$ and $P_{2}$ the corresponding projections of $\ell_{\infty}(\Upsilon)$ onto $\ell_{\infty}\left(\Upsilon_{1}\right)$ and $\ell_{\infty}\left(\Upsilon_{2}\right)$, respectively. Moreover, we shall choose a bijection $\varphi: \Upsilon_{2} \rightarrow \Upsilon$; we then obtain an isometric isomorphism $C_{\varphi}$ of $\ell_{\infty}(\Upsilon)$ onto the subspace $\ell_{\infty}\left(\Upsilon_{2}\right)$ by the definition $C_{\varphi} f=f \circ \varphi$ for each $f \in \ell_{\infty}(\Upsilon)$.

Let $E=X \oplus \ell_{\infty}(\Upsilon)$ with norm $\|(x, f)\|_{E}=\max \left\{\|x\|_{X},\|f\|_{\infty}\right\}$. We identify operators $T$ on $E$ with $(2 \times 2)$-matrices

$$
\left(\begin{array}{cc}
T_{1,1}: X \rightarrow X & T_{1,2}: \ell_{\infty}(\Upsilon) \rightarrow X \\
T_{2,1}: X \rightarrow \ell_{\infty}(\Upsilon) & T_{2,2}: \ell_{\infty}(\Upsilon) \rightarrow \ell_{\infty}(\Upsilon)
\end{array}\right)
$$

Note that assumption (II) and Lemma 7.1(ii) imply that the operator $T_{1,2}$ is always weakly compact. This fact will play a key role for us.

Despite our focus on left ideals, our first result about the Banach space $E$ is concerned with two-sided ideals.

Proposition 7.2. (i) The set

$$
\mathscr{W}_{1}=\left\{\left(\begin{array}{ll}
T_{1,1} & T_{1,2} \\
T_{2,1} & T_{2,2}
\end{array}\right) \in \mathscr{B}(E): T_{1,1} \in \mathscr{W}(X)\right\}
$$

is a proper, closed two-sided ideal of $\mathscr{B}(E)$, and $\mathscr{W}_{1}$ is a maximal two-sided ideal of $\mathscr{B}(E)$ if and only if $\mathscr{W}(X)$ is a maximal two-sided ideal of $\mathscr{B}(X)$.

(ii) The set

$$
\mathscr{W}_{2}=\left\{\left(\begin{array}{ll}
T_{1,1} & T_{1,2} \\
T_{2,1} & T_{2,2}
\end{array}\right) \in \mathscr{B}(E): T_{2,2} \in \mathscr{W}\left(\ell_{\infty}(\Upsilon)\right)\right\}
$$

is a proper, closed two-sided ideal of $\mathscr{B}(E)$, and the following three conditions are equivalent:

(a) $\mathscr{W}_{2}$ is a maximal two-sided ideal of $\mathscr{B}(E)$;

(b) $\mathscr{W}\left(\ell_{\infty}(\Upsilon)\right)$ is a maximal two-sided ideal of $\mathscr{B}\left(\ell_{\infty}(\Upsilon)\right)$;

(c) $\Upsilon$ is countable.

Proof. (i). The mapping

$$
T \mapsto T_{1,1}+\mathscr{W}(X), \quad \mathscr{B}(E) \rightarrow \mathscr{B}(X) / \mathscr{W}(X),
$$

is a surjective algebra homomorphism of norm one. Hence its kernel, which is equal to $\mathscr{W}_{1}$, is a closed two-sided ideal of $\mathscr{B}(E)$. This ideal is proper because, by assumption (I), $X$ is nonreflexive. The fundamental isomorphism theorem implies that $\mathscr{B}(E) / \mathscr{W}_{1} \cong \mathscr{B}(X) / \mathscr{W}(X)$. Thus the algebra $\mathscr{B}(E) / \mathscr{W}_{1}$ is simple if and only if $\mathscr{B}(X) / \mathscr{W}(X)$ is simple, and therefore $\mathscr{W}_{1}$ is a maximal two-sided ideal of $\mathscr{B}(E)$ if and only if $\mathscr{W}(X)$ is a maximal two-sided ideal of $\mathscr{B}(X)$.

(ii). An obvious modification of the argument given above shows that $\mathscr{W}_{2}$ is a proper, closed two-sided ideal of $\mathscr{B}(E)$, and that conditions (a) and (b) are equivalent. The implication $(\mathrm{c}) \Rightarrow(\mathrm{b})$ follows from [31, Proposition 2.f.4]. 
Conversely, to prove that $(\mathrm{b}) \Rightarrow(\mathrm{c})$, suppose that $\mathscr{W}\left(\ell_{\infty}(\Upsilon)\right)$ is a maximal two-sided ideal of $\mathscr{B}\left(\ell_{\infty}(\Upsilon)\right)$, and denote by $\mathscr{G}_{\ell_{\infty}}\left(\ell_{\infty}(\Upsilon)\right)$ the set of operators on $\ell_{\infty}(\Upsilon)$ that factor through $\ell_{\infty}$. This is a two-sided ideal of $\mathscr{B}\left(\ell_{\infty}(\Upsilon)\right)$ because $\ell_{\infty}$ is isomorphic to $\ell_{\infty} \oplus \ell_{\infty}$. Hence $\mathscr{G}_{\ell_{\infty}}\left(\ell_{\infty}(\Upsilon)\right)+\mathscr{W}\left(\ell_{\infty}(\Upsilon)\right)$ is also a two-sided ideal, which is strictly greater than $\mathscr{W}\left(\ell_{\infty}(\Upsilon)\right)$ because $\ell_{\infty}(\Upsilon)$ contains a complemented copy of $\ell_{\infty}$, and any projection with range isomorphic to $\ell_{\infty}$ belongs to $\mathscr{G}_{\ell_{\infty}}\left(\ell_{\infty}(\Upsilon)\right) \backslash \mathscr{W}\left(\ell_{\infty}(\Upsilon)\right)$. Consequently, by the maximality of $\mathscr{W}\left(\ell_{\infty}(\Upsilon)\right)$, there are operators $R \in \mathscr{G}_{\ell_{\infty}}\left(\ell_{\infty}(\Upsilon)\right)$ and $S \in \mathscr{W}\left(\ell_{\infty}(\Upsilon)\right)$ such that $I_{\ell_{\infty}(\Upsilon)}=R+S$. Then $R=I_{\ell_{\infty}(\Upsilon)}-S$ is a Fredholm operator by (2.5) and Lemma 7.1(i), so that $I_{\ell_{\infty}(\Upsilon)}=U R T$ for some operators $T, U \in \mathscr{B}\left(\ell_{\infty}(\Upsilon)\right)$ because $\ell_{\infty}(\Upsilon)$ is isomorphic to its hyperplanes. Thus the identity operator on $\ell_{\infty}(\Upsilon)$ factors through $\ell_{\infty}$, which is possible only if $\Upsilon$ is countable.

Set

$$
L=\left(\begin{array}{cc}
0 & 0 \\
V \kappa_{X} & C_{\varphi}
\end{array}\right) \in \mathscr{B}(E),
$$

where the operators $V$ and $C_{\varphi}$ were introduced on p. 27. Since the ranges of $V$ and $C_{\varphi}$ are contained in the complementary subspaces $\ell_{\infty}\left(\Upsilon_{1}\right)$ and $\ell_{\infty}\left(\Upsilon_{2}\right)$ of $\ell_{\infty}(\Upsilon)$, respectively, we have

$$
\begin{aligned}
\|L(x, f)\|_{E}=\left\|V \kappa_{X} x+C_{\varphi} f\right\|_{\infty} & =\max \left\{\left\|V \kappa_{X} x\right\|_{\infty},\left\|C_{\varphi} f\right\|_{\infty}\right\} \\
& =\max \left\{\left\|V \kappa_{X} x\right\|_{\infty},\|f\|_{\infty}\right\} \quad\left(x \in X, f \in \ell_{\infty}(\Upsilon)\right),
\end{aligned}
$$

which shows that the operator $L$ is bounded below because $V \kappa_{X}$ is bounded below. This conclusion is also immediate from our next result and Lemma 5.1.

Theorem 7.3. The ideal $\mathscr{W}_{1}$ defined in Proposition $7.2(\mathrm{i})$ is the left ideal generated by the operator L given by (7.1); that is,

$$
\mathscr{W}_{1}=\mathscr{L}_{\{L\}} .
$$

Proof. We have $L \in \mathscr{W}_{1}$ because $L_{1,1}=0$, and hence the inclusion $\supseteq$ follows.

We shall prove the reverse inclusion in three steps. First, we see that

$$
\left(\begin{array}{cc}
0 & 0 \\
0 & I_{\ell_{\infty}(\Upsilon)}
\end{array}\right)=\left(\begin{array}{cc}
0 & 0 \\
0 & C_{\varphi}^{-1} P_{2}
\end{array}\right)\left(\begin{array}{cc}
0 & 0 \\
V \kappa_{X} & C_{\varphi}
\end{array}\right) \in \mathscr{L}_{\{L\}},
$$

and consequently, for each $T_{1,2} \in \mathscr{B}\left(\ell_{\infty}(\Upsilon), X\right)$ and $T_{2,2} \in \mathscr{B}\left(\ell_{\infty}(\Upsilon)\right)$, we have

$$
\left(\begin{array}{cc}
0 & T_{1,2} \\
0 & T_{2,2}
\end{array}\right)=\left(\begin{array}{cc}
0 & T_{1,2} \\
0 & T_{2,2}
\end{array}\right)\left(\begin{array}{cc}
0 & 0 \\
0 & I_{\ell_{\infty}(\Upsilon)}
\end{array}\right) \in \mathscr{L}_{\{L\}}
$$

Second, let $T_{2,1} \in \mathscr{B}\left(X, \ell_{\infty}(\Upsilon)\right)$. Being bounded below, the operator $V \kappa_{X}$ is an isomorphism onto its range $Y:=V \kappa_{X}(X) \subseteq \ell_{\infty}\left(\Upsilon_{1}\right)$, so that it has an inverse $R \in \mathscr{B}(Y, X)$. By the injectivity of $\ell_{\infty}\left(\Upsilon_{1}\right)$, the composite operator $T_{2,1} R \in \mathscr{B}\left(Y, \ell_{\infty}(\Upsilon)\right)$ extends to an operator $S \in \mathscr{B}\left(\ell_{\infty}\left(\Upsilon_{1}\right), \ell_{\infty}(\Upsilon)\right)$, which then satisfies $S V \kappa_{X}=T_{2,1}$. Hence we have

$$
\left(\begin{array}{cc}
0 & 0 \\
T_{2,1} & 0
\end{array}\right)=\left(\begin{array}{cc}
0 & 0 \\
0 & S P_{1}
\end{array}\right)\left(\begin{array}{cc}
0 & 0 \\
V \kappa_{X} & C_{\varphi}
\end{array}\right) \in \mathscr{L}_{\{L\}}
$$


Third, each operator $T_{1,1} \in \mathscr{W}(X)$ satisfies $T_{1,1}^{* *}\left(X^{* *}\right) \subseteq \kappa_{X}(X)$ (e.g., see [34, Theorem 3.5.8]). We can therefore define an operator $U \in \mathscr{B}\left(\ell_{\infty}\left(\Upsilon_{1}\right), X\right)$ by $U f=\kappa_{X}^{-1} T_{1,1}^{* *} V^{-1} f$ for each $f \in \ell_{\infty}\left(\Upsilon_{1}\right)$. Since $\kappa_{X} U V \kappa_{X}=T_{1,1}^{* *} \kappa_{X}=\kappa_{X} T_{1,1}$, we have $U V \kappa_{X}=T_{1,1}$, and so

$$
\left(\begin{array}{cc}
T_{1,1} & 0 \\
0 & 0
\end{array}\right)=\left(\begin{array}{cc}
0 & U P_{1} \\
0 & 0
\end{array}\right)\left(\begin{array}{cc}
0 & 0 \\
V \kappa_{X} & C_{\varphi}
\end{array}\right) \in \mathscr{L}_{\{L\}}
$$

Combining (7.2)-(7.4), we conclude that each operator $T \in \mathscr{W}_{1}$ belongs to $\mathscr{L}_{\{L\}}$.

Remark 7.4. Since the operator $L$ given by (7.1) is bounded below and generates a proper left ideal of $\mathscr{B}(E)$, its range is not complemented in $E$ by Lemma 5.2. This is also easy to verify directly.

A Banach space $F$ has very few operators if $F$ is infinite-dimensional and each operator on $F$ is the sum of a scalar multiple of the identity operator and a compact operator; that is, $\mathscr{B}(F)=\mathbb{C} I_{F}+\mathscr{K}(F)$. Argyros and Haydon [4] constructed the first example of a Banach space $X_{\mathrm{AH}}$ which has very few operators. We shall now specialize to the case where $X=X_{\mathrm{AH}}$. The following result collects those properties of $X_{\mathrm{AH}}$ that we shall require.

Theorem 7.5 (Argyros and Haydon). There is a Banach space $X_{\mathrm{AH}}$ with the following three properties:

(i) $X_{\mathrm{AH}}$ has very few operators;

(ii) $X_{\mathrm{AH}}$ has a Schauder basis;

(iii) the dual space of $X_{\mathrm{AH}}$ is isomorphic to $\ell_{1}$.

Using this, we can easily prove Theorem 1.5.

Proof of Theorem 1.5. We begin by checking that $X_{\mathrm{AH}}$ satisfies the two assumptions made on p. 27: Theorem 7.5(iii) ensures that $X_{\mathrm{AH}}^{* *}$ is isomorphic to $\ell_{\infty}$, while Theorem 7.5(ii) (or (iii)) implies that $X_{\mathrm{AH}}$ does not contain $\ell_{\infty}$. Moreover, we have $\mathscr{W}\left(X_{\mathrm{AH}}\right)=\mathscr{K}\left(X_{\mathrm{AH}}\right)$ because Theorem 7.5(iii) implies that $X_{\mathrm{AH}}$ is non-reflexive, so that $\mathscr{W}\left(X_{\mathrm{AH}}\right)$ is a closed, non-zero, proper two-sided ideal of $\mathscr{B}\left(X_{\mathrm{AH}}\right)$, and $\mathscr{K}\left(X_{\mathrm{AH}}\right)$ is the only such ideal by Theorem 7.5(i)-(ii). Hence the set $\mathscr{K}_{1}$ given by (1.2) is equal to the ideal $\mathscr{W}_{1}$ defined in Proposition 7.2(i), and $\mathscr{W}_{1}$ is singly generated as a left ideal by Theorem 7.3. Theorem 7.5(i) implies that $\mathscr{K}_{1}$ has codimension one in $\mathscr{B}(E)$, so that it is trivially maximal as a left, right, and two-sided ideal. (The latter also follows from Proposition 7.2(i).) Being a non-zero two-sided ideal, $\mathscr{K}_{1}$ contains $\mathscr{F}(E)$, and therefore $\mathscr{K}_{1}$ is not fixed.

Remark 7.6. Theorem 1.5 implies that the class of Banach spaces for which Question (II) has a positive answer is not closed under finite direct sums. Indeed, $X_{\mathrm{AH}}$ and $\ell_{\infty}$ both belong to this class by Theorem 6.2 and Example 5.5(ii), respectively, whereas their direct sum does not.

We shall next give a characterization of the ideal $\mathscr{K}_{1}$ defined by (1.2). Theorem 1.6 will be an easy consequence of this result.

Theorem 7.7. Let $E=X_{\mathrm{AH}} \oplus \ell_{\infty}$. Then the following three conditions are equivalent for each subset $\mathscr{L}$ of $\mathscr{B}(E)$ : 
(a) $\mathscr{L}=\mathscr{K}_{1}$

(b) $\mathscr{L}$ is a non-fixed, finitely-generated, maximal left ideal of $\mathscr{B}(E)$;

(c) $\mathscr{L}$ is a maximal left ideal of $\mathscr{B}(E)$ and contains an operator which is bounded below.

Proof. $(\mathrm{a}) \Rightarrow(\mathrm{b})$. This is immediate from Theorem 1.5.

(b) $\Rightarrow($ c). Suppose that $\mathscr{L}$ is a non-fixed, finitely-generated, maximal left ideal of $\mathscr{B}(E)$, so that $\mathscr{L}=\mathscr{L}_{\Gamma}$ for some non-empty, finite subset $\Gamma$ of $\mathscr{B}(E)$. Set $n=|\Gamma| \in \mathbb{N}$. By Lemma 5.1, the operator $\Psi_{\Gamma}$ is bounded below. Moreover, there is an operator $T \in \mathscr{B}\left(E^{n}, E\right)$ which is bounded below because $X_{\mathrm{AH}}$ embeds in $\ell_{\infty}$, and $\ell_{\infty}$ is isomorphic to the direct sum of $2 n-1$ copies of itself. Hence the composite operator $T \Psi_{\Gamma}$ is bounded below, and it belongs to $\mathscr{L}$ by $(2.8)$.

(c) $\Rightarrow($ a). Suppose that $\mathscr{L}$ is a maximal left ideal of $\mathscr{B}(E)$ and that $\mathscr{L}$ contains an operator $R=\left(R_{j, k}\right)_{j, k=1}^{2}$ which is bounded below. Then $R$ does not belong to any fixed maximal left ideal, so that $\mathscr{E}(E) \subseteq \mathscr{L}$ by Corollary 4.2. Lemma 7.1 shows that each operator from $\ell_{\infty}$ to $X_{\mathrm{AH}}$ is strictly singular, and thus inessential. Hence, by [15, Proposition 1], each operator from $X_{\mathrm{AH}}$ to $\ell_{\infty}$ is also inessential, and so we conclude that

$$
\begin{aligned}
\left\{\left(\begin{array}{ll}
T_{1,1} & T_{1,2} \\
T_{2,1} & T_{2,2}
\end{array}\right): T_{1,1} \in \mathscr{K}\left(X_{\mathrm{AH}}\right), T_{1,2} \in \mathscr{B}\left(\ell_{\infty}, X_{\mathrm{AH}}\right),\right. \\
\left.T_{2,1} \in \mathscr{B}\left(X_{\mathrm{AH}}, \ell_{\infty}\right), T_{2,2} \in \mathscr{W}\left(\ell_{\infty}\right)\right\}=\mathscr{E}(E) \subseteq \mathscr{L} .
\end{aligned}
$$

Since the operator $R$ is bounded below, its restriction $\left.R\right|_{\ell_{\infty}}=\left(\begin{array}{l}R_{1,2} \\ R_{2,2}\end{array}\right)$ is also bounded below, and is thus an upper semi-Fredholm operator. Consequently $\left(\begin{array}{c}0 \\ R_{2,2}\end{array}\right)$ is an upper semiFredholm operator by (2.6) because $R_{1,2}$ is strictly singular, and therefore $R_{2,2}$ is an upper semi-Fredholm operator. Let $Q \in \mathscr{F}\left(\ell_{\infty}\right)$ be a projection onto ker $R_{2,2}$. Then the restriction of $R_{2,2}$ to ker $Q$ is an isomorphism onto its range, which is a closed subspace of $\ell_{\infty}$. Since $\ell_{\infty}$ is injective, the inverse of this isomorphism extends to an operator $S: \ell_{\infty} \rightarrow \operatorname{ker} Q \subseteq \ell_{\infty}$, which then satisfies $S R_{2,2}=I_{\ell_{\infty}}-Q$. Hence we have

$$
\left(\begin{array}{cc}
0 & 0 \\
S R_{2,1} & I_{\ell_{\infty}}-Q
\end{array}\right)=\left(\begin{array}{cc}
0 & 0 \\
0 & S
\end{array}\right)\left(\begin{array}{ll}
R_{1,1} & R_{1,2} \\
R_{2,1} & R_{2,2}
\end{array}\right) \in \mathscr{L}, \quad \text { and thus } \quad\left(\begin{array}{cc}
0 & 0 \\
0 & I_{\ell_{\infty}}
\end{array}\right) \in \mathscr{L}
$$

by (7.5). Combining this with (7.5), we see that $\mathscr{K}_{1} \subseteq \mathscr{L}$, and consequently $\mathscr{K}_{1}=\mathscr{L}$ by the maximality of $\mathscr{K}_{1}$.

Proof of Theorem 1.6. The equivalence of conditions (a) and (b) in Theorem 7.7 shows that $\mathscr{K}_{1}$ is the unique non-fixed, finitely-generated, maximal left ideal of $\mathscr{B}(E)$. Proposition 7.2(ii) implies that $\mathscr{W}_{2}$ is a maximal two-sided ideal. Since $\mathscr{F}(E) \subseteq \mathscr{W}_{2} \nsubseteq \mathscr{K}_{1}$, $\mathscr{W}_{2}$ is not contained in any finitely-generated, maximal left ideal of $\mathscr{B}(E)$.

One may wonder whether the conclusion of Theorem 1.5 that the ideal $\mathscr{W}_{1}$ introduced in Proposition 7.2(i) is maximal as a left ideal might be true more generally, that is, not only in the case where $X$ is Argyros-Haydon's Banach space. Our next result implies that this 
is false for $X=c_{0}$. Note that all weakly compact operators on $c_{0}$ are compact, so that, in this case, $\mathscr{W}_{1}$ is equal to

$$
\mathscr{K}_{1}=\left\{\left(\begin{array}{ll}
T_{1,1} & T_{1,2} \\
T_{2,1} & T_{2,2}
\end{array}\right) \in \mathscr{B}\left(c_{0} \oplus \ell_{\infty}\right): T_{1,1} \in \mathscr{K}\left(c_{0}\right)\right\} .
$$

Proposition 7.8. The ideal $\mathscr{K}_{1}$ given by (7.6) is not contained in any finitely-generated, maximal left ideal of $\mathscr{B}\left(c_{0} \oplus \ell_{\infty}\right)$.

For clarity, we present the main technical step in the proof of Proposition 7.8 as a separate lemma.

Lemma 7.9. For each operator $T \in \mathscr{B}\left(c_{0}\right) \backslash \Phi_{+}\left(c_{0}\right)$, there exist a projection $Q_{0} \in \mathscr{B}\left(c_{0}\right)$ and a normalized basic sequence $\left(x_{n}\right)_{n \in \mathbb{N}}$ in $c_{0}$ such that $\left(x_{n}\right)_{n \in \mathbb{N}}$ is equivalent to the standard unit vector basis for $c_{0}$ and

$$
Q_{0} x_{2 n-1}=x_{2 n-1}, \quad Q_{0} x_{2 n}=0, \quad \text { and } \quad\left\|T x_{n}\right\| \leqslant \frac{1}{n} \quad(n \in \mathbb{N}) .
$$

Proof. Let $\left(e_{n}\right)_{n \in \mathbb{N}}$ denote the standard unit vector basis for $c_{0}$. Since $T \notin \Phi_{+}\left(c_{0}\right)$, there are two cases to consider.

Case 1: $\alpha(T)=\infty$. Then $\operatorname{ker} T$ contains a closed subspace $Y$ which is isomorphic to $c_{0}$ and complemented in $c_{0}$ (e.g., see [31, Proposition 2.a.2]). Let $\left(x_{n}\right)_{n \in \mathbb{N}}$ be a normalized Schauder basis for $Y$ such that $\left(x_{n}\right)_{n \in \mathbb{N}}$ is equivalent to $\left(e_{n}\right)_{n \in \mathbb{N}}$. Since $Y$ is complemented in $c_{0}$ and the basis $\left(x_{n}\right)_{n \in \mathbb{N}}$ is unconditional, there is a projection $Q_{0} \in \mathscr{B}\left(c_{0}\right)$ which satisfies the first two identities in (7.7), while the third one is trivial because $x_{n} \in Y \subseteq \operatorname{ker} T$ for each $n \in \mathbb{N}$.

Case 2: $\alpha(T)<\infty$ and $T\left(c_{0}\right)$ is not closed. For each $n \in \mathbb{N}$, choose $\varepsilon_{n} \in(0,1)$ such that $(1+\|T\|) \varepsilon_{n}\left(1-\varepsilon_{n}\right)^{-1} \leqslant n^{-1}$. By induction, we shall construct a normalized block basic sequence $\left(x_{n}\right)_{n \in \mathbb{N}}$ of $\left(e_{n}\right)_{n \in \mathbb{N}}$ such that $\left\|T x_{n}\right\| \leqslant n^{-1}$ for each $n \in \mathbb{N}$.

To start the induction, we observe that $T$ cannot be bounded below because its range is not closed, so that we can find a unit vector $y_{1} \in c_{0}$ such that $\left\|T y_{1}\right\| \leqslant \varepsilon_{1}$. Approximating $y_{1}$ within $\varepsilon_{1}$ by a finitely-supported vector and normalizing it, we obtain a finitely-supported unit vector $x_{1} \in c_{0}$ such that $\left\|T x_{1}\right\| \leqslant(1+\|T\|) \varepsilon_{1}\left(1-\varepsilon_{1}\right)^{-1} \leqslant 1$ by the choice of $\varepsilon_{1}$.

Now assume inductively that, for some $n \in \mathbb{N}$, unit vectors $x_{1}, \ldots, x_{n} \in c_{0}$ with consecutive supports have been chosen such that $\left\|T x_{j}\right\| \leqslant 1 / j$ for each $j \in\{1, \ldots, n\}$. Let $m \in \mathbb{N}$ be the maximum of the support of $x_{n}$, so that $x_{1}, \ldots, x_{n} \in \operatorname{span}\left\{e_{1}, \ldots, e_{m}\right\}$, and let $P_{m}$ be the $m^{\text {th }}$ basis projection associated with $\left(e_{j}\right)_{j \in \mathbb{N}}$. If $\left.T\right|_{\text {ker } P_{m}}$ were bounded below, then it would have closed range, so that

$$
T\left(c_{0}\right)=T\left(\operatorname{ker} P_{m}\right)+\operatorname{span}\left\{T e_{1}, \ldots, T e_{m}\right\}
$$

would also be closed, being the sum of a closed subspace and a finite-dimensional one. This is false, and hence $\left.T\right|_{\operatorname{ker} P_{m}}$ is not bounded below. We can therefore choose a unit vector $y_{n+1} \in \operatorname{ker} P_{m}$ such that $\left\|T y_{n+1}\right\| \leqslant \varepsilon_{n+1}$. Now, as in the first step of the induction, we approximate $y_{n+1}$ within $\varepsilon_{n+1}$ by a finitely-supported vector in ker $P_{m}$ and normalize it 
to obtain a finitely-supported unit vector $x_{n+1} \in \operatorname{ker} P_{m}$ such that

$$
\left\|T x_{n+1}\right\| \leqslant \frac{(1+\|T\|) \varepsilon_{n+1}}{1-\varepsilon_{n+1}} \leqslant \frac{1}{n+1}
$$

by the choice of $\varepsilon_{n+1}$. Hence the induction continues.

By [31, Proposition 2.a.1], the sequence $\left(x_{n}\right)_{n \in \mathbb{N}}$ is equivalent to $\left(e_{n}\right)_{n \in \mathbb{N}}$, and its closed linear span is complemented in $c_{0}$. Therefore, as in Case 1 , we obtain a projection $Q_{0} \in \mathscr{B}\left(c_{0}\right)$ such that the first two identities in (7.7) are satisfied, while the third one holds by the construction of $\left(x_{n}\right)_{n \in \mathbb{N}}$.

Proof of Proposition 7.8. Assume towards a contradiction that $\mathscr{L}$ is a finitely-generated, maximal left ideal of $\mathscr{B}\left(c_{0} \oplus \ell_{\infty}\right)$ such that $\mathscr{K}_{1} \subseteq \mathscr{L}$. Proposition 2.1 implies that $\mathscr{L}$ is generated by a single operator, say

$$
T=\left(\begin{array}{ll}
T_{1,1} & T_{1,2} \\
T_{2,1} & T_{2,2}
\end{array}\right) \in \mathscr{B}\left(c_{0} \oplus \ell_{\infty}\right) .
$$

We claim that $T_{1,1}$ is not an upper semi-Fredholm operator. Assume the contrary; that is, $\operatorname{ker} T_{1,1}$ is finite-dimensional, so that we can take a projection $P \in \mathscr{F}\left(c_{0}\right)$ onto $\operatorname{ker} T_{1,1}$, and $T_{1,1}\left(c_{0}\right)$ is closed. Then the restriction $\widetilde{T}_{1,1}: x \mapsto T_{1,1} x, \operatorname{ker} P \rightarrow T_{1,1}\left(c_{0}\right)$, is an isomorphism. Its range is complemented in $c_{0}$ by Sobczyk's theorem [43] because it is isomorphic to ker $P$, which is a closed subspace of finite codimension in $c_{0}$, and hence isomorphic to $c_{0}$. We can therefore extend the inverse of $\widetilde{T}_{1,1}$ to an operator $S \in \mathscr{B}\left(c_{0}\right)$, which then satisfies $S T_{1,1}=I_{c_{0}}-P$. Since $P$ has finite rank, we have

$$
\left(\begin{array}{cc}
P & 0 \\
0 & I_{\ell_{\infty}}
\end{array}\right) \in \mathscr{K}_{1} \subseteq \mathscr{L} \quad \text { and } \quad\left(\begin{array}{cc}
T_{1,1} & 0 \\
0 & 0
\end{array}\right)=T-\left(\begin{array}{cc}
0 & T_{1,2} \\
T_{2,1} & T_{2,2}
\end{array}\right) \in \mathscr{L}-\mathscr{K}_{1} \subseteq \mathscr{L},
$$

which implies that

$$
\left(\begin{array}{cc}
I_{c_{0}} & 0 \\
0 & I_{\ell_{\infty}}
\end{array}\right)=\left(\begin{array}{cc}
P & 0 \\
0 & I_{\ell_{\infty}}
\end{array}\right)+\left(\begin{array}{cc}
S & 0 \\
0 & 0
\end{array}\right)\left(\begin{array}{cc}
T_{1,1} & 0 \\
0 & 0
\end{array}\right) \in \mathscr{L}
$$

This, however, contradicts the assumption that the left ideal $\mathscr{L}$ is proper, and thus completes the proof of our claim.

Hence, by Lemma 7.9, we obtain a projection $Q_{0} \in \mathscr{B}\left(c_{0}\right)$ and a normalized basic sequence $\left(x_{n}\right)_{n \in \mathbb{N}}$ in $c_{0}$ such that $\left(x_{n}\right)_{n \in \mathbb{N}}$ is equivalent to the standard unit vector basis $\left(e_{n}\right)_{n \in \mathbb{N}}$ for $c_{0}$ and

$$
Q_{0} x_{2 n-1}=x_{2 n-1}, \quad Q_{0} x_{2 n}=0, \quad \text { and } \quad\left\|T_{1,1} x_{n}\right\| \leqslant \frac{1}{n} \quad(n \in \mathbb{N}) .
$$

The sequence $\left(x_{n}\right)_{n \in \mathbb{N}}$ is weakly null because it is equivalent to the weakly null sequence $\left(e_{n}\right)_{n \in \mathbb{N}}$, and so the sequence $\left(R x_{n}\right)_{n \in \mathbb{N}}$ is norm-null for each $R \in \mathscr{K}\left(c_{0}\right)$. Now let

$$
Q=\left(\begin{array}{cc}
Q_{0} & 0 \\
0 & 0
\end{array}\right) \in \mathscr{B}\left(c_{0} \oplus \ell_{\infty}\right)
$$

The maximality of the left ideal $\mathscr{L}$ implies that either
(i) $Q \in \mathscr{L}$
or
(ii) $\mathscr{L}+\mathscr{L}_{\{Q\}}=\mathscr{B}\left(c_{0} \oplus \ell_{\infty}\right)$. 
We shall complete the proof by showing that both of these alternatives are impossible.

In case (i), we can write $Q=S T$ for some operator $S=\left(S_{j, k}\right)_{j, k=1}^{2} \in \mathscr{B}\left(c_{0} \oplus \ell_{\infty}\right)$. Defining $P_{0} \in \mathscr{B}\left(c_{0} \oplus \ell_{\infty}, c_{0}\right)$ by $P_{0}(x, f)=x$ for each $x \in c_{0}$ and $f \in \ell_{\infty}$, we have

$$
x_{2 n-1}=P_{0} Q\left(x_{2 n-1}, 0\right)=P_{0} S T\left(x_{2 n-1}, 0\right)=S_{1,1} T_{1,1} x_{2 n-1}+S_{1,2} T_{2,1} x_{2 n-1} \quad(n \in \mathbb{N}) .
$$

This, however, is absurd since the left-hand side is a unit vector, whereas the right-hand side norm-converges to 0 as $n \rightarrow \infty$ because $\left\|T_{1,1} x_{2 n-1}\right\| \rightarrow 0$ by (7.8) and $S_{1,2} T_{2,1} \in$ $\mathscr{W}\left(c_{0}\right)=\mathscr{K}\left(c_{0}\right)$.

In case (ii), there are operators $U, V \in \mathscr{B}\left(c_{0} \oplus \ell_{\infty}\right)$ such that $I_{c_{0} \oplus \ell_{\infty}}=U T+V Q$. Define $P_{0}$ as above, and write $U=\left(U_{j, k}\right)_{j, k=1}^{2}$. Then, since $Q_{0} x_{2 n}=0$, we have

$$
x_{2 n}=P_{0}(U T+V Q)\left(x_{2 n}, 0\right)=U_{1,1} T_{1,1} x_{2 n}+U_{1,2} T_{2,1} x_{2 n} \quad(n \in \mathbb{N}),
$$

which leads to a contradiction as in case (i) because the left-hand side is a unit vector, whereas the right-hand side norm-converges to 0 as $n \rightarrow \infty$.

\section{ACKNOWLEDGEMENTS}

We are grateful to David Blecher (Houston) and Mikael Rørdam (Copenhagen) for having informed us that the conjecture of the first author and Żelazko stated on p. 4 has a positive answer for $C^{*}$-algebras, and for having shown us how to deduce it from standard facts.

Key parts of this work were carried out during a visit of Piotr Koszmider to Lancaster in February 2012. The visit was supported by a London Mathematical Society Scheme 2 grant (ref. 21101). The authors gratefully acknowledge this support.

\section{REFERENCES}

[1] F. Albiac and N. J. Kalton, Topics in Banach space theory, Graduate Texts Math. 233, SpringerVerlag, New York, 2006.

[2] D. Amir, Continuous function spaces with the bounded extension property, Bull. Res. Concil Israel Sect. F 101 (1962), 133-138.

[3] S. A. Argyros, J. F. Castillo, A. S. Granero, M. Jiménez, and J. P. Moreno, Complementation and embeddings of $c_{0}(I)$ in Banach spaces, Proc. London Math. Soc. 85 (2002), 742-768.

[4] S. A. Argyros and R. G. Haydon, A hereditarily indecomposable $\mathscr{L}_{\infty}$-space that solves the scalarplus-compact problem, Acta Math. 206 (2011), 1-54.

[5] J. W. Baker, Some uncomplemented subspaces of $C(X)$ of the type $C(Y)$, Studia Math. 36 (1970), $85-103$.

[6] D. Blecher and T. Kania, Finite generation in $C^{*}$-algebras and Hilbert $C^{*}$-modules, in preparation.

[7] N. Boudi, Banach algebras in which every left ideal is countably generated, Irish Math. Soc. Bull. 48 (2002), 17-24.

[8] W. W. Comfort and S. Negrepontis, The theory of ultrafilters, Grundlehren math. Wissenschaften 211, Springer-Verlag, New York-Heidelberg, 1974.

[9] H. G. Dales, Banach algebras and automatic continuity, London Math. Soc. Monographs 24, Clarendon Press, Oxford, 2000.

[10] H. G. Dales and W. Żelazko, Generators of maximal left ideals in Banach algebras, Studia Math. 212 (2012), 173-193.

[11] W. J. Davis, T. Figiel, W. B. Johnson, and A. Pełczyński, Factoring weakly compact operators, J. Funct. Anal. 17 (1974), 311-327. 
[12] I. S. Edelstein and B. S. Mityagin, Homotopy type of linear groups of two classes of Banach spaces, Funct. Anal. Appl. 4 (1970), 221-231.

[13] V. Ferreira and G. Tomassini, Finiteness properties of topological algebras, Ann. Scuola Norm. Sup. Pisa 5 (1978), 471-488.

[14] L. Gillman, Countably generated ideals in rings of continuous functions, Proc. Amer. Math. Soc. 11 (1960), 660-666.

[15] M. González, On essentially incomparable Banach spaces, Math. Z. 215 (1994), 621-629.

[16] W. T. Gowers and B. Maurey, The unconditional basic sequence problem, J. Amer. Math. Soc. 6 (1993), 851-874.

[17] B. Gramsch, Eine Idealstruktur Banachscher Operatoralgebren, J. Reine Angew. Math. 225 (1967), $97-115$.

[18] A. S. Granero, On complemented subspaces of $c_{0}(I)$, Atti Sem. Mat. Fis. Univ. Módena XLVI (1998), $35-36$.

[19] A. Grothendieck, Une caractérisation vectorielle métrique des espaces $L_{1}$, Canad. J. Math. 7 (1955), $552-561$.

[20] N. Grønbæk, Morita equivalence for Banach algebras, J. Pure Appl. Algebra 99 (1995), 183-219.

[21] N. Jacobson, Basic Algebra II, W. H. Freeman, San Francisco, California, 1980.

[22] T. Jech, Set Theory, Third Millennium Ed., revised and expanded, Springer-Verlag, Berlin, 2003.

[23] R. V. Kadison and J. R. Ringrose, Fundamentals of the theory of operator algebras I: Elementary theory, Pure and Applied Math. 100, Academic Press, New York, 1983.

[24] R. V. Kadison and J. R. Ringrose, Fundamentals of the theory of operator algebras II: Advanced theory, Pure and Applied Math. 100, Academic Press, Orlando, Florida, 1986.

[25] N. J. Kalton and N. T. Peck, Twisted sums of sequence spaces and the three space problem, Trans. Amer. Math. Soc. 255 (1979), 1-30.

[26] D. Kleinecke, Almost-finite, compact, and inessential operators, Proc. Amer. Math. Soc. 14 (1963), 863-868.

[27] P. Koszmider, Banach spaces of continuous functions with few operators, Math. Ann. 330 (2004), 151-183.

[28] N. J. Laustsen, Maximal ideals in the algebra of operators on certain Banach spaces, Proc. Edinburgh Math. Soc. 45 (2002), 523-546.

[29] N. J. Laustsen, Commutators of operators on Banach spaces, J. Operator Th. 48 (2002), 503-514.

[30] J. Lindenstrauss, On the extension property for compact operators, Bull. Amer. Math. Soc. 68 (1962), $484-487$.

[31] J. Lindenstrauss and L. Tzafriri, Classical Banach spaces I, Ergeb. Math. Grenzgeb. 92, SpringerVerlag, Berlin-New York, 1977.

[32] J. Lindenstrauss and L. Tzafriri, Classical Banach spaces II, Ergeb. Math. Grenzgeb. 97, SpringerVerlag, Berlin-New York, 1979.

[33] E. Luft, The two-sided closed ideals of the algebra of bounded linear operators of a Hilbert space, Czechoslovak Math. J. 18 (1968), 595-605.

[34] R. E. Megginson, An introduction to Banach space theory, Graduate Texts Math. 183, Springer-Verlag, New York, 1998.

[35] G. J. Murphy, $C^{*}$-algebras and operator theory, Academic Press, Boston, MA, 1990.

[36] A. Pełczyński, On strictly singular and strictly cosingular operators. I. Strictly singular and strictly cosingular operators in $C(S)$-spaces, Bull. Acad. Polon. Sci. Sér. Sci. Math. Astronom. Phys. 13 (1965), 31-36.

[37] G. Plebanek, A construction of a Banach space $C(K)$ with few operators, Topology Appl. 143 (2004), 217-239.

[38] B. Pospísili, On bicompact spaces. Publ. Fac. Sci. Univ. Masaryk 270 (1939), 3-16.

[39] R. T. Prosser, On the ideal structure of operator algebras, Mem. Amer. Math. Soc. 45 (1963). 
[40] A. Rosenberg, The number of irreducible representations of simple rings with no minimal ideals, Amer. J. Math, 75 (1953), 523-530.

[41] Th. Schlumprecht. An arbitrarily distortable Banach space. Israel J. Math. 76 (1991), 81-95.

[42] A. M. Sinclair and A. W. Tullo, Noetherian Banach algebras are finite dimensional, Math. Ann. 211 (1974), 151-153.

[43] A. Sobczyk, Projection of the space $(m)$ on its subspace (co), Bull. Amer. Math. Soc. 47 (1941), 938-947.

[44] A. Szankowski, $\mathscr{B}(H)$ does not have the approximation property, Acta Math. 147 (1981), 89-108.

Dales, Kania, and Laustsen

Department of Mathematics and Statistics, Fylde College

Lancaster University, Lancaster LA1 4YF, UK;

e-mail: g.dales@lancaster.ac.uk, t.kania@lancaster.ac.uk and

n.laustsen@lancaster.ac.uk

Kochanek

Institute of Mathematics, University of Silesia

ul. Bankowa 14, 40-007 Katowice, Poland;

e-mail: t_kochanek@wp.pl

Koszmider

Institute of Mathematics, Polish Academy of Sciences

ul. Śniadeckich 8, 00-956 Warszawa, Poland;

e-mail: P.Koszmider@Impan.pl 